\title{
Detailed Empirical Study of the Term Structure of Interest Rates. Emergence of Power Laws and Scaling Laws
}

\author{
Thomas Alderweireld ${ }^{1}$ and Jean Nuyts ${ }^{2}$
}

\begin{abstract}
The technique of Padé Approximants, introduced in a previous work, is applied to extended recent data on the distribution of variations of interest rates compiled by the Federal Reserve System in the US. It is shown that new power laws and new scaling laws emerge for any maturity not only as a function of the Lag but also as a function of the average inital rate. This is especially true for the one year maturity where critical forms and critical exponents are obtained. This suggests future work in the direction of constructing a theory of variations of interest rates at a more "microscopic" level.
\end{abstract}

\footnotetext{
${ }^{1}$ Thomas.Alderweireld@umh.ac.be, Université de Mons-Hainaut, 20 Place du Parc, 7000 Mons, Belgium

22Jean.Nuyts@umh.ac.be, Université de Mons-Hainaut, 20 Place du Parc, 7000 Mons, Belgium
} 


\section{Introduction}

Many models have been constructed to try to describe and/or predict the term structure of interest rates. Recently, it has been shown on a specific set of data that the empirical distribution of the variation of interest rates is rather well reproduced by using the technique of Padé Approximants [1. This article is a further step in this direction. The classical approach is based on Brownian motions of various kinds. It leads to exponential tails (or superposition of exponential tails) in the distributions. These so called narrow tails are usually badly rejected by the data. Levy-flight [2], 3], 44 distributions have also been proposed. In this case the tails of the distributions decrease as the inverse of a small power of the variation of the interest rates (see below (10) ), in fact a power comprised between one and two. The predicted tails are really too fat and are also rejected by the data. Sometimes rather unjustified cuts have been used to sharpen the Levy tails.

In a recent paper [1], analyzing numerically the data (Data $\{1\}$ ) on the American daily spot interest rates compounded by the Federal Reserve System [5] between February 15, 1977 and August 4, 1997 ( $N_{\text {events }}=5108$ corresponding to 5108 opening days), it was discovered that the fat tails, known to appear in the empirical distributions, decrease essentially as a fourth power of the variation of the interest rate and are thus naturally amenable to ratios of polynomials. It was shown that the empirical distributions, averaged on the initial interest rates, are very well fitted with simple Padé Approximants. More recently, articles taking into account the fat, but not too fat, tails and power laws have been proposed [6]-7].

In this paper, using more complete data (Data $\{2\}$ ) recently available, again from the FRS between January 2, 1962 and December 27, 2002 (10230 opening days, see (6) below), we have been able to study in a more detailed way the Padé fits. At a first stage, we confirmed the preceding analysis. The results are summarized in section 2. In section 3, we have refined the analysis by dividing the new data in suitable subsets, restricting ourselves to intervals in the initial interest rate. This has allowed us to confirm and to extend the validity of the preceding results, but also and more importantly to suggest new scaling laws.

These results will be used in a forthcoming article [8] to try to build a "microscopic model" of interest rates. 


\section{Padé Approximants fits. Update of the Previ- ous Results}

In this section, we present briefly the notation and update the results, which have been obtained in [1] for the Data $\{1\}$, using the full data set (Data $\{2\}$ ) available today [5].

Suppose that, at some initial time $t_{0}$, the interest rate (for some maturity $[\mathrm{m}]$ ) is $I_{0}$ and, at some later time $t_{f}$, it is $I_{f}$. The lag and the variation of interest rates were defined as follows

- The lag is the time interval between the initial and final times

$$
L=t_{f}-t_{0}
$$

It should be stressed that the lag is expressed in consecutive opening days and that non working days were simply discarded $[9]$.

- The variation of interest rates is

$$
v=I_{f}-I_{0} .
$$

Since, in the data, the interest rates are expressed exactly in basis points (0.01 percent), the basis point has been chosen as the natural unit. When discretized, the variables $I$ and $v$ are thus represented by integers, $\widehat{I}$ and $\widehat{v}$.

Let us denote by $p^{[m]}\left(L, v, I_{0}\right)$ the probability distribution and by $\widehat{p}^{[m]}\left(L, \widehat{v}, \widehat{I}_{0}\right)$ the corresponding discretized distribution. They are the probability that the interest rate for maturity $[m]$ has moved from $I_{0}$ to $I_{f}$ during the lag $L$. Their norms are

$$
\begin{aligned}
\int_{-\infty}^{\infty} p^{[m]}\left(L, v, I_{0}\right) d v & =1 \\
\sum_{\widehat{v}=-\infty}^{\infty} \widehat{p}^{[m]}\left(L, \widehat{v}, \widehat{I}_{0}\right) & =1 .
\end{aligned}
$$

In the FRS Data $\{1\}$ and $\{2\}$, the maturities $[m]=1,2,3,5,7,10$ and 30 years are given. All the daily interest rates are expressed exactly in basis points. They vary roughly from $1.5 \%=150$ basis points to $18 \%=1800$ basis points. The discrete empirical distributions $\widehat{w}(\widehat{I})$ of the initial interest rates, for a given maturity 
and for the two sets, are defined by

$$
\begin{aligned}
& \widehat{w}(\widehat{I})=\frac{N^{d}(\widehat{I})}{N_{\text {events }}}, \\
& N^{d}(\widehat{I})=\text { number of days the interest rate, for maturity }[m] \text {, } \\
& \text { was } \widehat{I} \text { basis points in the FRS Data }\{1\} \text { or }\{2\} \text {, } \\
& N_{\text {events }}=5108 \text { in Data }\{1\} \text { for all }[m] \text {, } \\
& \begin{array}{|c|c|c|c|c|c|c|c|c|}
\hline[m] & = & 1 & 2 & 3 & 5 & 7 & 10 & 30 \\
\hline N_{\text {events }}= & 10230 & 6606 & 10199 & 10199 & 8323 & 10199 & 6214 \\
\hline
\end{array}
\end{aligned}
$$

As an example, the FRS empirical distribution $\widehat{w}$ for the maturity $[m]=1$ year and for the Data $\{2\}$ is given in Figure (1). Obviously, we have for any such distribution

$$
\sum_{\widehat{I}=150}^{1800} \widehat{w}(\widehat{I})=1
$$

The main subject of study in [1] was in fact the discrete average $\widehat{p}_{\text {average }}^{[m]}(L, \widehat{v})$ defined by

$$
\widehat{p}_{\text {average }}^{[m]}(L, \widehat{v})=\sum_{\widehat{I_{0}}=150}^{1800} \widehat{w}^{[m]}\left(I_{0}\right) \widehat{p}^{[m]}\left(L, \widehat{v}, \widehat{I}_{0}\right) .
$$

The discretized averages can easily be extracted from the data for lags (in days) such that $1 \leq L \leq 30$ and for variations of interest rates $\widehat{v}$ (expressed in basis points) included in the range $R_{v}=[-200 \leq \widehat{v} \leq 200]$. Indeed, outside this range, the data is always zero. The discrete normalization becomes

$$
\sum_{\widehat{v}=-200}^{200} \widehat{p}_{\text {average }}^{[m]}(L, \widehat{v})=1 .
$$

It has been first shown empirically, using Data $\{1\}$, that the tail of the term structure of the interest rates decreases essentially as a fourth power of the variation of the interest rates i.e.

$$
p_{\text {average }}^{[m]}(L, v) \approx \kappa \frac{1}{|v|^{d}} \quad \text { when }|v| \text { becomes large }
$$

with a suitable constant $\kappa$ and a power $d$

$$
d \approx 4
$$


This means that the distributions decrease much faster than what Levy flight models suggest (namely $1 \leq d \leq 2$ ) but much more slowly than what Brownian models $p_{\text {average }} \approx \kappa \exp \left(-v^{2} / \sigma^{2}\right)$ predict.

Using detailed fits, it has been shown in [1] that the average distribution (8) can be very well (amazingly well) approximated by a Padé Approximant $P(0,4)$, i.e. with a constant numerator and a polynomial of fourth degree in the denominator. More precisely, for all maturities $[m]$ and for all lags $L$, the following Padé form of the probability distributions, in terms of $\widehat{v}$,

$$
\widehat{p}_{\text {average }}^{[m]}(L, \widehat{v})=\frac{q_{1}}{\pi\left(1+\left(q_{1}^{2}+2 q_{2}\right) \widehat{v}^{2}+q_{2}^{2} \widehat{v}^{4}\right)},
$$

fits the Data $\{2\}$ (and the Data $\{1\}$ set) rather well.

Let us enumerate a few of the properties of this form and of the two remaining real parameters $q_{1}$ and $q_{2}$ which depend on the lag $L$ and on the maturity $[m]$.

1. The continuous distribution corresponding to (12) is, for $q_{1}$ positive, a purely positive real function. In fact it is constructed as the modulus square of the complex function $f$

$$
\begin{aligned}
f_{\text {average }}^{[m]}(L, v) & =\frac{\sqrt{q_{1}}}{\sqrt{\pi}\left(1+i q_{1} v+q_{2} v^{2}\right)}, \\
p_{\text {average }}^{[m]}(L, v) & =\left|f_{\text {average }}^{[m]}(L, v)\right|^{2} .
\end{aligned}
$$

2. The normalization (3) of the distribution (14) is automatic by construction.

3. The distribution depends only on $v^{2}$, hence it is symmetrical under the interchange $\{v \leftrightarrow-v\}$. This symmetry was checked empirically to hold to a very precise degree. In other words, violations of this symmetry are zero well within one standard deviation.

4. The tail of the distribution (12) is of the form $v^{-4}$. The Bayesian Hill estimators [10] of the empirical tails favor an approximate decrease of this kind.

5. An estimate of $q_{1}$ is given by

$$
q_{1}=\pi \widehat{p}_{\text {average }}^{[m]}(L, 0)
$$

and of $q_{2}$ by

$$
q_{2}=-\sigma^{-1}
$$

where $\sigma$ stands for the variance of the data distribution. 
6. The parameters $q_{1}$ and $q_{2}$ have very simple regular forms in term of the lag $L$ when $L$ varies from one day to about a month and for any maturity $[m]$. Essentially

$$
q_{i} \approx \beta_{i}\left(\frac{L}{L_{\mathrm{r}}}\right)^{-\mu_{i}} \quad, \quad i=1,2
$$

where, for convenience, we have chosen one arbitrary reference value $L_{\mathrm{r}}=$ 15 days. The $\mu_{i}$, which we call scaling parameters, and the less essential multiplication constants $\beta_{i}$ depend on $[m]$ but not on $L$. The Table (11) (resp. Table (2)) shows the $\mu_{i}$ and the ratio $\mu_{2} / \mu_{1}$ that we have obtained for the 7 investigated maturities using the Data $\{2\}$ (resp. Data $\{1\}$ ) set.

In other words, as illustrated by the Figures (2) and (3) for $[\mathrm{m}]=2$ years, as an example, in a $\ln \left(q_{i}\right)$ versus $\ln (L)$ plot the data points align along a straight line with slope $-\mu_{i}$. The plots for the other $[m]$ 's follow analogous straight lines.

7. Moreover, one may note that one critical exponent is approximatively related to the other exponent by (see Tables (11) (2))

$$
\mu_{2} \approx 1.8 \mu_{1},
$$

8. It should be stressed that this scaling law (17) seems to be an important discovery [1] which had escaped attention.

\section{Padé Approximants fits. Initial Interest Rate Dependence}

More recently, we have studied the extended set of data (Data $\{2\}$ ) and performed a more refined analysis.

Indeed, the new data having often a higher statistics, meaningful subsets of data can be defined with initial interest rates limited to intervals. We have found that subsets containing about two thousand events are large enough to lead to a sufficiently precise determination of the parameters.

A detailed numerical analysis of the distributions restricting the data to an almost fixed initial interest rate $I_{0}$ has convinced us that the same Padé behavior prevails. An approximate determination of the dependence of the Padé parameters $q_{i}$ on initial interest rate $I_{0}$ follows. It suggests that new scaling laws are at work.

Let us summarize our results 
1. We have first divided the Data $\{2\}$ set in subsets where $I_{0}$ is restricted to regions (overlapping or not)

$$
\Delta=\left[\widehat{I}_{0 \min }, \widehat{I}_{0 \max }\right] .
$$

The total probability $\omega_{\Delta}$ to be inside $\Delta$ and the total numbers of events $N_{\Delta}$ inside $\Delta$ are given by

$$
\begin{aligned}
\omega_{\Delta} & =\sum_{\widehat{I}=\widehat{I}_{0 \min }}^{\widehat{I}_{\max }} \widehat{w}(\widehat{I}), \\
N_{\Delta} & =\omega_{\Delta} N_{\text {events }} .
\end{aligned}
$$

In the present analysis, the regions have be chosen in such a way that the $N_{\Delta}$ 's are around two thousands. If the number of events is chosen to be smaller than about two thousand, the accuracy in the determination of the parameters becomes hazardous. On the other hand, there seems to be no need to select larger regions with more events in them. The events in Data $\{2\}$ can thus, for example for $[m]=1$ year, be separated in five non overlapping intervals covering the full $\widehat{I}_{0}$ range.

2. Let us define $\overline{I_{0}}$ as the empirical average of $\widehat{I}$ in a given region $\Delta$

$$
\overline{I_{0}}=\frac{\sum_{\widehat{I}=\widehat{I}_{\min }}^{\widehat{I}_{\max }} \widehat{w}(\widehat{I}) \widehat{I}}{\omega_{\Delta}} .
$$

3. The distributions, for the events within a region $\Delta$ (see (24) for a precise definition), are then supposed, in first approximation, to be represented by normalized (3) Padé forms

$$
\bar{p}\left(L, \widehat{v}, \overline{I_{0}}\right)=\frac{q_{1}}{\pi\left(1+\left(q_{1}^{2}+2 q_{2}\right) \widehat{v}^{2}+q_{2}^{2} \widehat{v}^{4}\right)} .
$$

4. The parameters $q_{i}$ appearing in Eq.(23) (for a given maturity $[m]$ ) are thus evaluated as functions of $L$ and $\overline{I_{0}}$. They are obtained from the data by minimizing a $\chi^{2}$ function. In about all instances the fits are very good as exemplified by the $\chi^{2}$ values.

5. In Figure (44), as an example using the Data $\{2\}$ with $L=1$ and $[m]=1$ year, two thousand points in the region $\Delta=[420$ basis points, 553 basis points $]$ 
(see (19)) have been selected and the empirical points are plotted versus the best Padé fit. The average initial interest rate (22) is about $\overline{I_{0}}=502$ basis points. In this case, the values of $q_{i}$ evaluated in their respective units are $q_{1}=38.6 \pm 1.3(\%)^{-1}$ and $q_{2}=-308 \pm 19(\%)^{-2}$. The obtained $\chi^{2}$ is 61 with 83 degrees of freedom. In almost all $L,[\mathrm{~m}]$ cases an eye check of the curves is as satisfactory and the computed $\chi^{2}$ are as good.

6. At this point, an important remark is worth making. It should be stressed that the parameters $q_{i}$ obtained this way are not really those at $I_{0}=\overline{I_{0}}$ itself but a complicated average of the approximate $q_{i}$ in the corresponding $\Delta$ range. Indeed, it is obvious that the superposition of two or more Pade forms is, at best, only approximatively of a Padé form. Since this is an important point, let us explain it more precisely. If $\widehat{p}(L, \widehat{v}, \widehat{I})$ is known for any $\widehat{I}$, then the average probability $\bar{p}\left(L, \widehat{v}, \overline{I_{0}}\right)(\underline{23})$ on $\Delta$ is defined by

$$
\bar{p}\left(L, \widehat{v}, \overline{I_{0}}\right)=\frac{\sum_{\widehat{I}=\widehat{I}_{0 \min }}^{\widehat{I}_{0 \max }} \widehat{w}(\widehat{I}) \widehat{p}(L, \widehat{v}, \widehat{I})}{\omega_{\Delta}} .
$$

If it then were true that $\widehat{p}(L, \widehat{v}, \widehat{I})$ is exactly of the Padé form (which is anyhow not a correct statement but, at best, an approximate one), the $\Delta$-average probability $\bar{p}\left(L, \widehat{v}, \overline{I_{0}}\right)$ would not exactly be of a Padé form.

7. After the fits have been performed, we find that the new parameters $q_{i}$ depend effectively and importantly on $\overline{I_{0}}$.

8. In a first approach, we have chosen to minimize the natural $\chi^{2}$ leaving $q_{1}$ as free parameter and by determining $q_{2}$ from the experimental variance using the relation (16). The interested reader can find the precise definition of the $\chi^{2}$ in the reference [1], in and after their Eq.(22).

9. We defer to the next section a detailed discussion of the parameters $q_{i}$, obtained from the data, as functions of the lag $L$ and of the average interest rate $\bar{I}_{0}$.

\section{Padé parameters as a function of the lag, of the maturity and, of the initial interest rate}

In this section, we summarize the scaling law and the $\bar{I}_{0}$ dependence that are suggested by the empirical data. 


\subsection{General considerations and scaling laws}

The first scaling law and the $I_{0}$ dependence can be summarized as follows. For each maturity, the empirical $q_{i}$ are given by the approximate forms (compare to (17))

$$
q_{i} \approx \beta_{i}\left(\frac{L}{L_{\mathrm{r}}}\right)^{-\mu_{i}} \mathcal{F}_{i}\left(\overline{\frac{I_{0}}{I_{r}}}\right) .
$$

where the $\mathcal{F}_{i}$ are functions depending of the initial interest rate. For convenience, we have chosen two arbitrary reference values

$$
\begin{aligned}
L_{\mathrm{r}} & =15 \text { days } \\
I_{\mathrm{r}} & =600 \text { basis points } .
\end{aligned}
$$

In (25), the lag $L$ is in days, the average initial rate $\bar{I}_{0}$ is in basis points, the parameters $q_{1}$ and $\beta_{1}$ are in inverse basis points, the parameters $q_{2}$ and $\beta_{2}$ are in inverse basis points squared while the scaling parameters $\mu_{i}$ are pure numbers.

The scaling law (25) says that, for every maturity $[m]$, each of the parameters $q_{i}$ is the product of two functions. One function depends on the lag $L$ only and has the form of a power law as (17). The second function, $\mathcal{F}_{i}$, depends on $\bar{I}_{0}$ only.

The best values for the parameters $\mu_{i}$ are given in Table 1 as a function of the maturity. The form of the $\mathcal{F}_{i}$ can be found in the fourteen plots given in Figures (5) (8)). In Figure (5) , for $[m]=1$ year and $L=1$ day, the $\ln \left(q_{1}\right)$ is plotted versus $\bar{I}_{0} / I_{r}$. In Figure (6), for $[m]=1$ year and $L=1$ day, the $\ln \left(q_{2}\right)$ is plotted versus $\bar{I}_{0} / I_{r}$. In Figure (17) there are six plots where for $L=1, \ln \left(q_{1}\right)$ is given versus $\bar{I}_{0} / I_{r}$ for $[m]=$ 2, 3, 5, 7, 10, 30 years. Finally, in Figure (8) there are six plots where for $L=1$, $\ln \left(q_{2}\right)$ is given versus $\bar{I}_{0} / I_{r}$ for $[m]=2,3,5,7,10,30$ years.

We have shown that the assumption in (25) that the $q_{i}$ factorize (separation of variables) is favoured by the data. Hence, for a given maturity, the distributions of $\ln \left(q_{i}\right)$ as a function of $\bar{I}_{0}$ for different $L$ values just differ by a constant shift. This shift on the $\ln \left(q_{i}\right)$ axis is equal to

$$
\ln \left(q_{i}\right)-\ln \left(q_{i}^{\prime}\right)=\mu_{i} \ln \left(L^{\prime} / L\right) .
$$

To illustrate this, the Figures (9) and (10) show the distributions of $\ln \left(q_{i}\right)$, for $[m]=5$ years as a function of $\bar{I}_{0}$ for 3 different $L$ values (1, 10 and 30 days). To make the comparison easier, the $L=10$ days and the $L=30$ days distributions have been moved upwards by their respective shift term $\left(\mu_{i} \ln (10)\right.$ and $\mu_{i} \ln (30)$ respectively). For the other maturity, one obtains similar plots.

It has to be emphasized that this variable separation in the approximate forms may be a hint of a scaling law which is at work. 


\subsection{The scaling laws for the $[m]=1$ year case}

For the $[m]>1$ year data, no particular simple shape for the function $\mathcal{F}_{i}$ can be extracted. In the $[m]=1$ year data, the functions $\mathcal{F}_{i}$ have a very simple shape. The

$\ln \left(q_{i}\right)$ distributions as function of $\overline{I_{0}}$ (see Figures (15) (6) for $L=1$ day) can be fitted with straight lines and the following extremely simple approximate forms hold

$$
q_{i} \approx \beta_{i}\left(\frac{L}{L_{\mathrm{r}}}\right)^{-\mu_{i}} \exp \left(\left(\frac{\overline{I_{0}}}{I_{r}}\right)^{-\nu_{i}}\right)
$$

where

$$
\begin{gathered}
\ln \left(\beta_{1}\right)=3.14 \pm 0.02, \quad \mu_{1}=0.657 \pm 0.005, \quad \nu_{1}=1.32 \pm 0.02 \\
\ln \left(\beta_{2}\right)=4.51 \pm 0.02, \quad \mu_{2}=1.197 \pm 0.005, \quad \nu_{2}=2.19 \pm 0.02
\end{gathered}
$$

Hence, for the particular case of $[m]=1$ year, we obtain an interplay of two simple scaling laws with critical exponents $\mu_{i}$ and $\nu_{i}$.

\section{Conclusion}

In this paper, we have extended an earlier work aimed at approximating the term structure of interest rates by ratios of polynomials called Padé Approximants. The form which is used is a purely positive Padé $P[0,4]$ with a constant numerator and a fourth degree denominator in the variation of interest rates.

We have shown that for any maturity, for any lag and for initial interest rates restricted to regions, the form holds to a very good degree of approximation, better than what should have been hoped a priori.

The empirical parameters are represented by functions which point toward the existence of scaling laws and scaling exponents at a more "microscopic level". These scaling laws were already discovered and discussed [1] in terms the lag variable. In this paper, using the extended FRS data [5] now available, we have shown that scaling laws seem also to be at work not only in the lag, as was shown previously, but also in the initial interest rate. This is true especially for $[m]=1$ year maturity where simple critical forms (28) appear both in the lag $L$ and in the average interest rate $\bar{I}_{0}$ and critical exponents can be extracted.

In a forthcoming paper, we will try to use this result and the scaling laws which are suggested by the data to build a theory of the term structure of interest rates at a "microscopic level". 


\section{Acknowledgment}

The work of one author (T.A.) was supported in part by Belgian I.I.S.N. (Institut Interuniversitaire des Sciences Nucléaires). The work of the other (J.N.) was supported in part by the Belgian F.N.R.S. (Fonds National de la Recherche Scientifique). He would also like to thank Pr. Isabelle Platten for discussions and numerical simulations in an early phase of this research. 


\section{Figure Caption}

Figure 1.

The FRS empirical weight distribution $\widehat{w}$ of the interest rates (see (5) ) for the maturity $[m]=1$ year and for the Data set $\{2\}$.

Figure 2.

Distribution of $\ln \left(q_{1}\right)$ as a function of $\ln (L / 15)$ for $[m]=2$ years using Data set $\{2\}$.

Figure 3.

Distribution of $\ln \left(q_{2}\right)$ as a function of $\ln (L / 15)$ for $[m]=2$ years using Data set $\{2\}$.

Figure 4 .

One example of a fit to the Data set $\{2\}$ for $[m]=1$ year and $L=1$ day by a Padé Approximant (continuous curve). The chosen subset (see (19)) corresponds to 2000 points in the region $I_{0 \min }=420$ and $I_{0 \max }=553$. The $\bar{I}_{0}$ is computed to be 502 basis points. The empirical points are plotted versus the best Padé fit. In this case, the values of $q_{i}$ evaluated in their respective units are $q_{1}=38.6 \pm 1.3(\%)^{-1}$ and $q_{2}=-308 \pm 19(\%)^{-2}$. The obtained $\chi^{2}$ is 61 with 83 degrees of freedom.

Figure 5 .

The parameter $\ln \left(q_{1}\right)$ as a function of $\overline{I_{0}} / 600$ for $[m]=1$ year and $L=1$ day. These points have been obtained by minimizing the $\chi^{2}$ as a function of parameter $q_{1}$. Each point corresponds to a subset of data containing close to two thousand points with average $I_{0}$ value equal to $\overline{I_{0}}$. The subsets are overlapping so that the points are not independent. The unit used for $q_{1}$ is $(\%)^{-1}$. For $\overline{I_{0}}$ it is the basis point. The straight line is a linear fit. Its equation is $\ln \left(q_{1}\right)=\ln \left(\beta_{1}\right)-\nu_{1}\left(\overline{I_{0}} / 600\right)-\mu_{1} \ln (1 / 15)$ with $\nu_{1}=1.32 \pm 0.02$ and $\ln \left(\beta_{1}\right)=3.14 \pm 0.02$.

Figure 6. 
The parameter $\ln \left(q_{2}\right)$ as a function of $\overline{I_{0}} / 600$ for $[m]=1$ year and $L=1$ day. These points have been obtained using the relation defined in (16). Each point corresponds to a subset of data containing close to two thousand points with average $I_{0}$ value equal to $\overline{I_{0}}$. The subsets are overlapping so that the points are not independent. The error on $\ln \left(q_{2}\right)$ is computed by propagating the error on the estimate of the variance. The unit used for $q_{2}$ is $(\%)^{-2}$. For $\overline{I_{0}}$ it is the basis point. The straight line is a linear fit. Its equation is $\ln \left(q_{2}\right)=$ $\ln \left(\beta_{2}\right)-\nu_{2}\left(\overline{I_{0}} / 600\right)-\mu_{2} \ln (1 / 15)$ with $\nu_{2}=2.19 \pm 0.02$ and $\ln \left(\beta_{2}\right)=4.51 \pm 0.02$.

\section{Figure 7.}

The parameter $\ln \left(q_{1}\right)$ as a function of $\overline{I_{0}} / 600$ for $[m]=2,3,5,7,10,30$ years and $L=1$ day. These points have been obtained by minimizing the $\chi^{2}$ as a function of parameter $q_{1}$. Each point corresponds to a subset of data containing close to two thousand points with average $I_{0}$ value equal to $\overline{I_{0}}$. The subsets are overlapping so that the points are not independent. The unit used for $q_{1}$ is $(\%)^{-1}$. For $\overline{I_{0}}$ it is the basis point.

\section{Figure 8 .}

The parameter $\ln \left(q_{2}\right)$ as a function of $\overline{I_{0}} / 600$ for $[m]=2,3,5,7,10,30$ years and $L=1$ day. These points have been obtained using the relation (16). Each point corresponds to a subset of data containing close to two thousand points with average $I_{0}$ value equal to $\overline{I_{0}}$. The subsets are overlapping so that the points are not independent. The unit used for $q_{2}$ is $(\%)^{-2}$. For $\overline{I_{0}}$ it is the basis point. The error on $\ln \left(q_{2}\right)$ is computed by propagating the error on the estimate of the variance.

Figure 9.

Distribution of $\ln \left(q_{1}\right)$, for $[m]=5$ years as a function of $\overline{I_{0}} / 600$ for 3 different $L$ values (1,10 and 30 days) where the $L=10$ and 30 days distributions have been shifted on the $\ln \left(q_{1}\right)$ axis by the correcting term (27).

Figure 10.

Distribution of $\ln \left(q_{2}\right)$, for $[m]=5$ years as a function of $\overline{I_{0}} / 600$ for 3 different $L$ values (1,10 and 30 days) where the $L=10$ and 30 days distributions have been shifted on the $\ln \left(q_{2}\right)$ axis by the correcting term (27). 


\section{Table Caption}

Table 1.

The values of the best fits to the parameters $\mu_{i}$ (see (25)) using Data set $\{2\}$. These parameters are given as a function of the maturity $[m]$. The standard errors correspond to a unit deviation in the $\chi^{2}$.

Table 2.

The values of the best fits to the parameters $\mu_{i}$ (see (25)) using Data set $\{1\}$. These parameters are given as a function of the maturity $[m]$. The standard errors correspond to a unit deviation in the $\chi^{2}$. 
Figure (11)

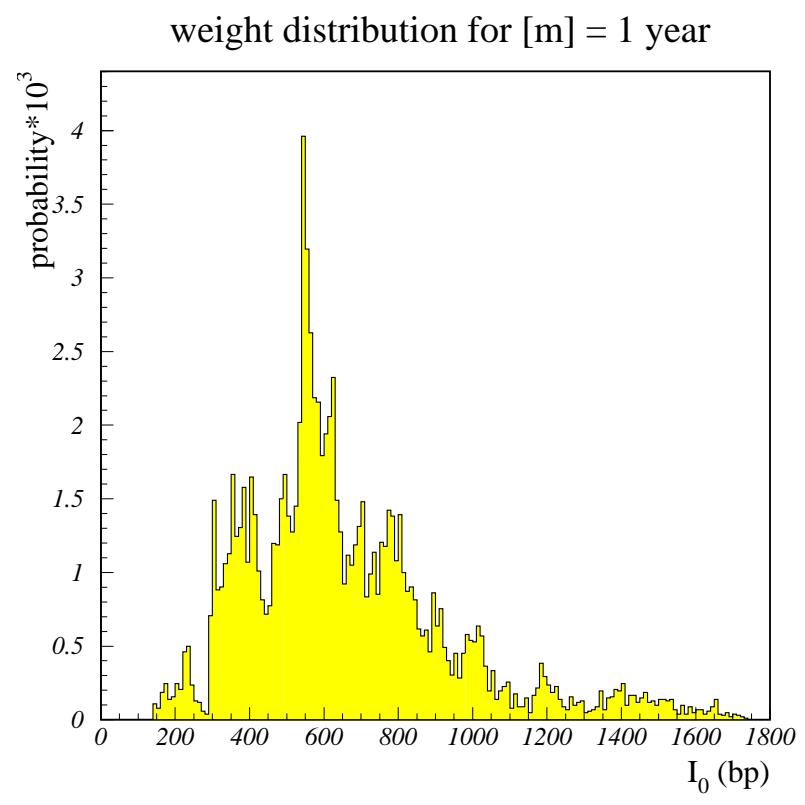

Figure (2)

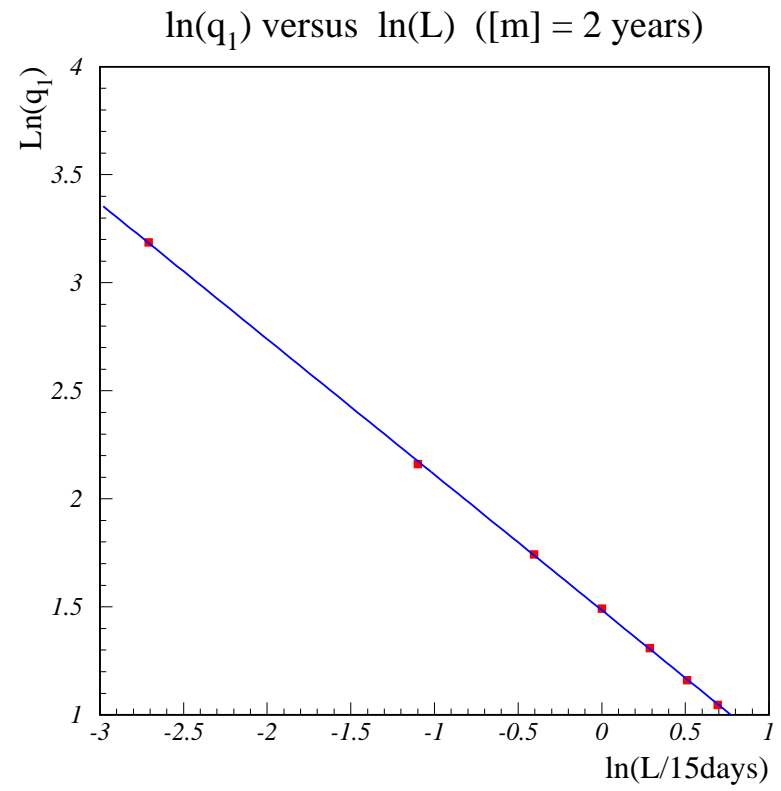


Figure (3)

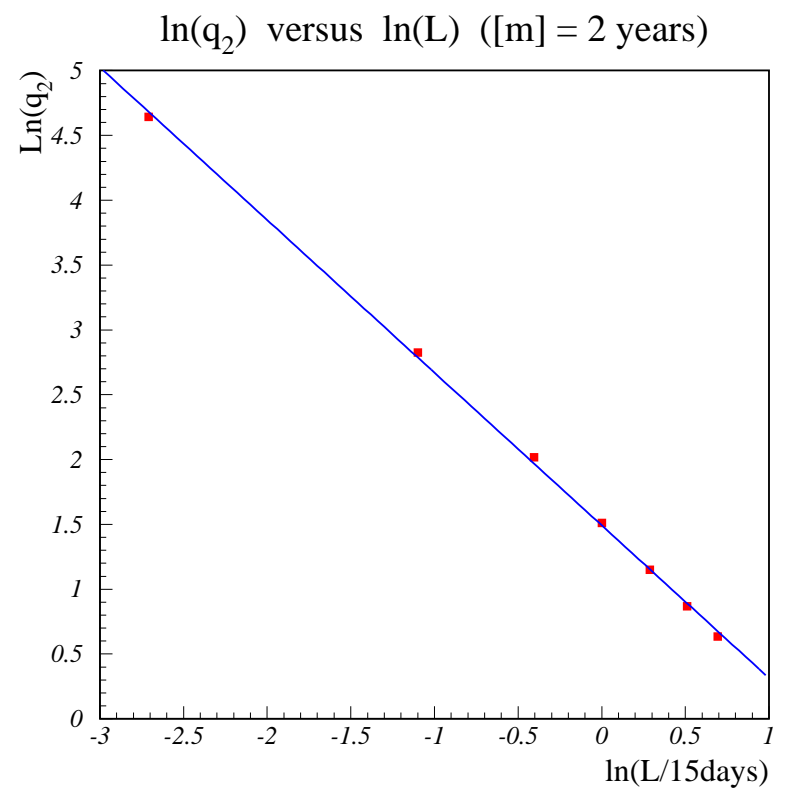

Figure (4)

A fit for $[\mathrm{m}]=1$ year, $\mathrm{L}=1$ day and average $\mathrm{I}_{0}=502 \mathrm{bp}$

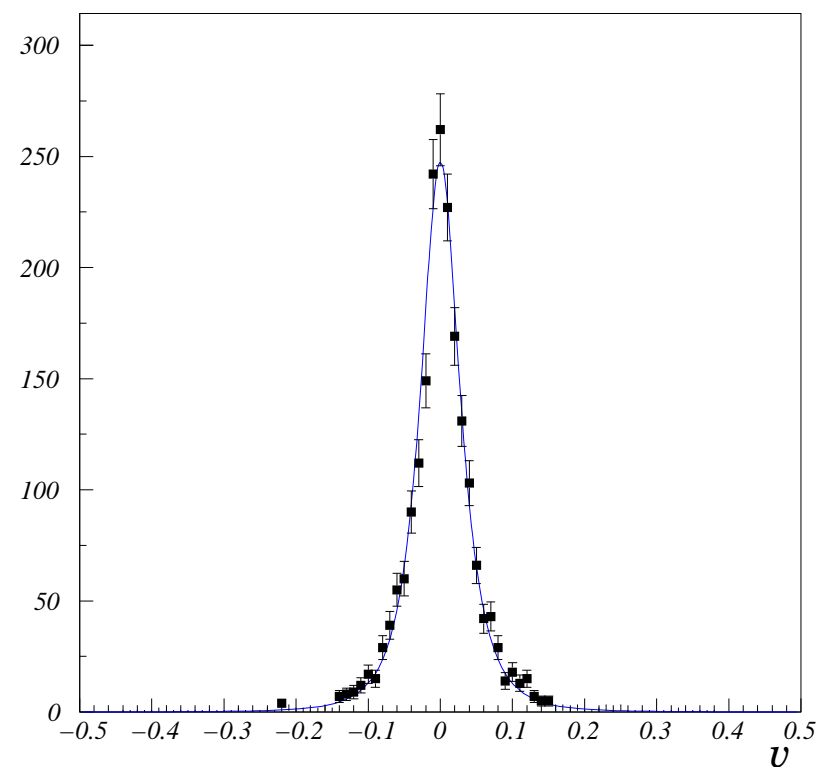


Figure (5)

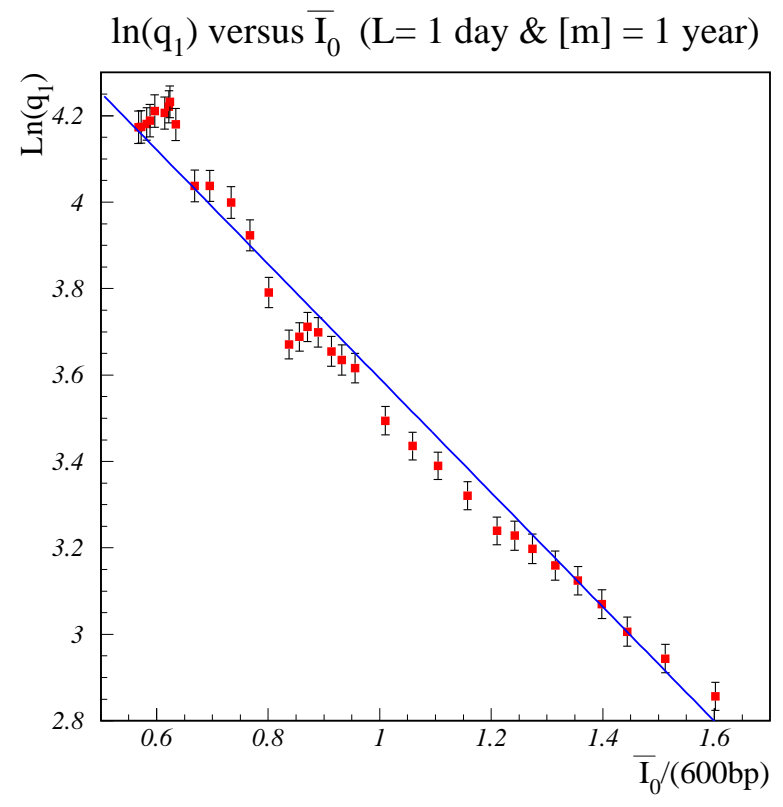

Figure (6)

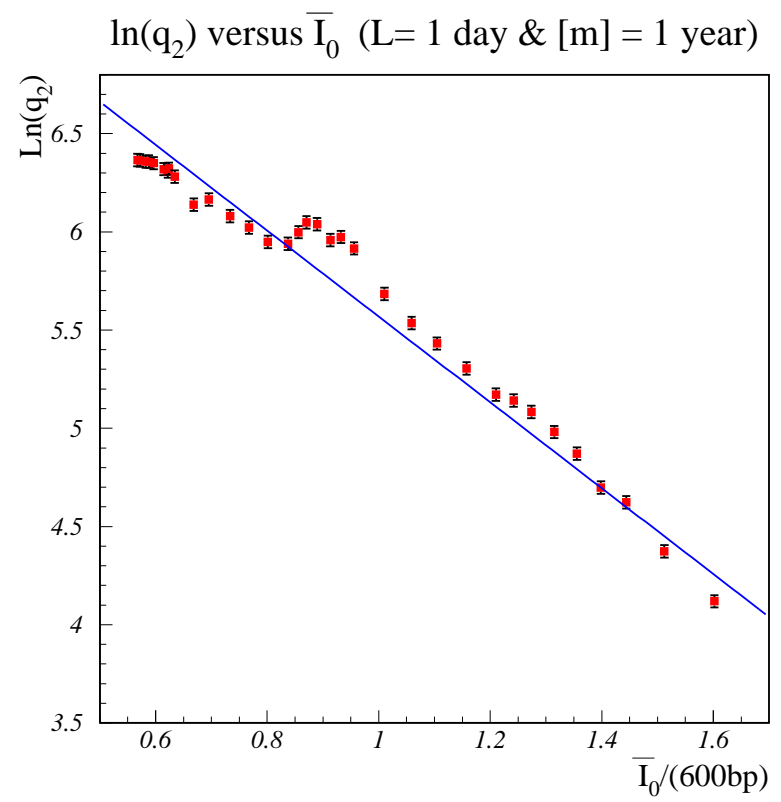


Figure (7)

$\ln \left(\mathrm{q}_{1}\right)$ versus $\overline{\mathrm{I}}_{0}(\mathrm{~L}=1$ day $\&[\mathrm{~m}]=2$ years $)$
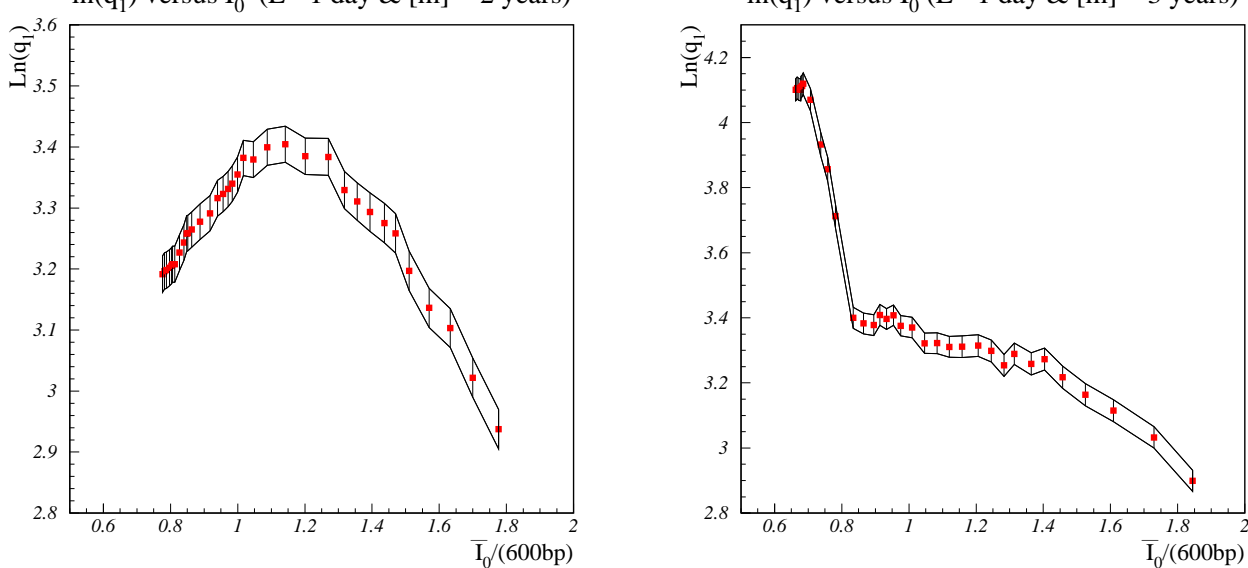

$\ln \left(\mathrm{q}_{1}\right)$ versus $\overline{\mathrm{I}}_{0}(\mathrm{~L}=1$ day $\&[\mathrm{~m}]=5$ years $)$
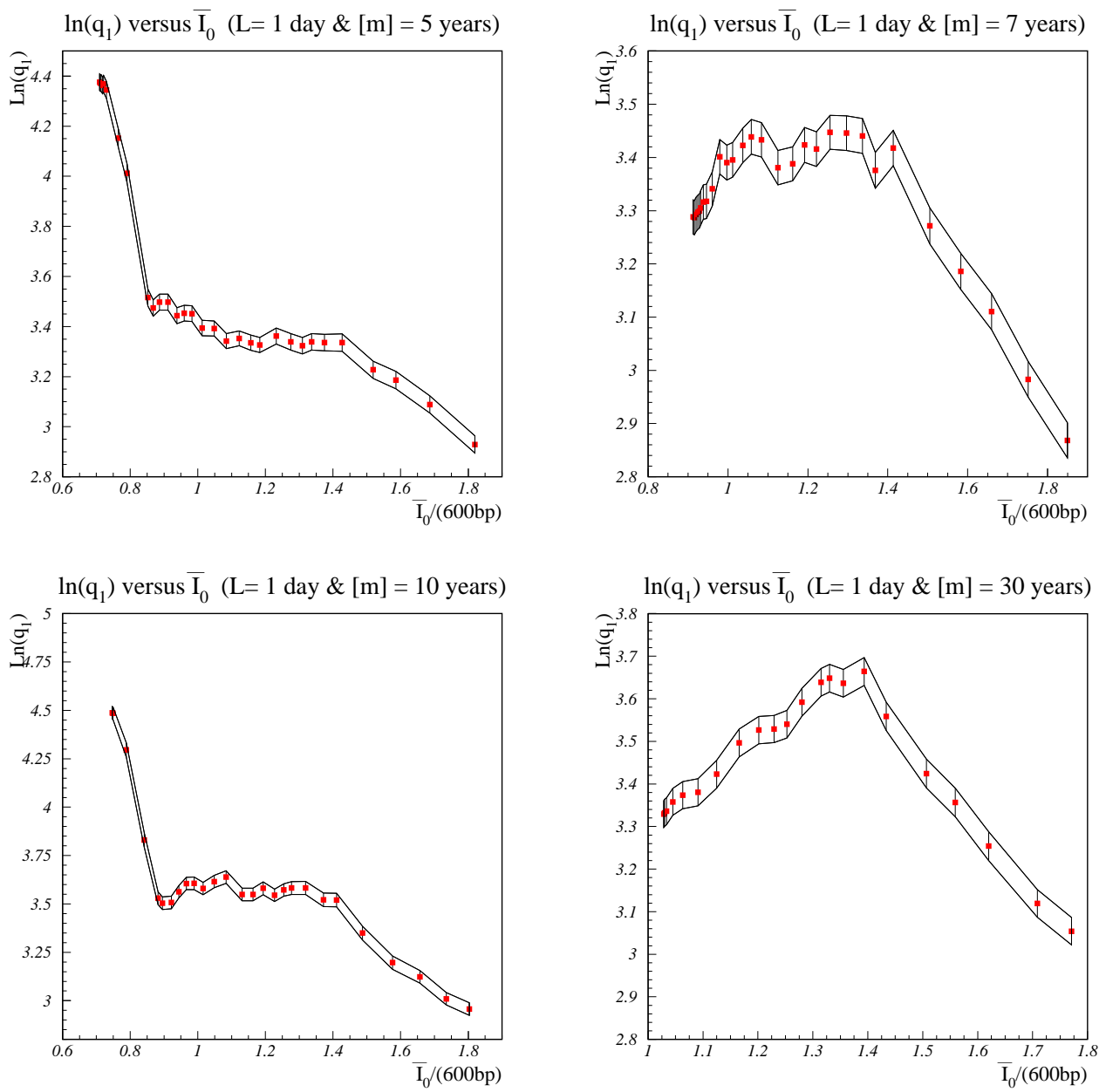
Figure (8)

$\ln \left(\mathrm{q}_{2}\right)$ versus $\overline{\mathrm{I}}_{0}(\mathrm{~L}=1$ day $\&[\mathrm{~m}]=2$ years $)$
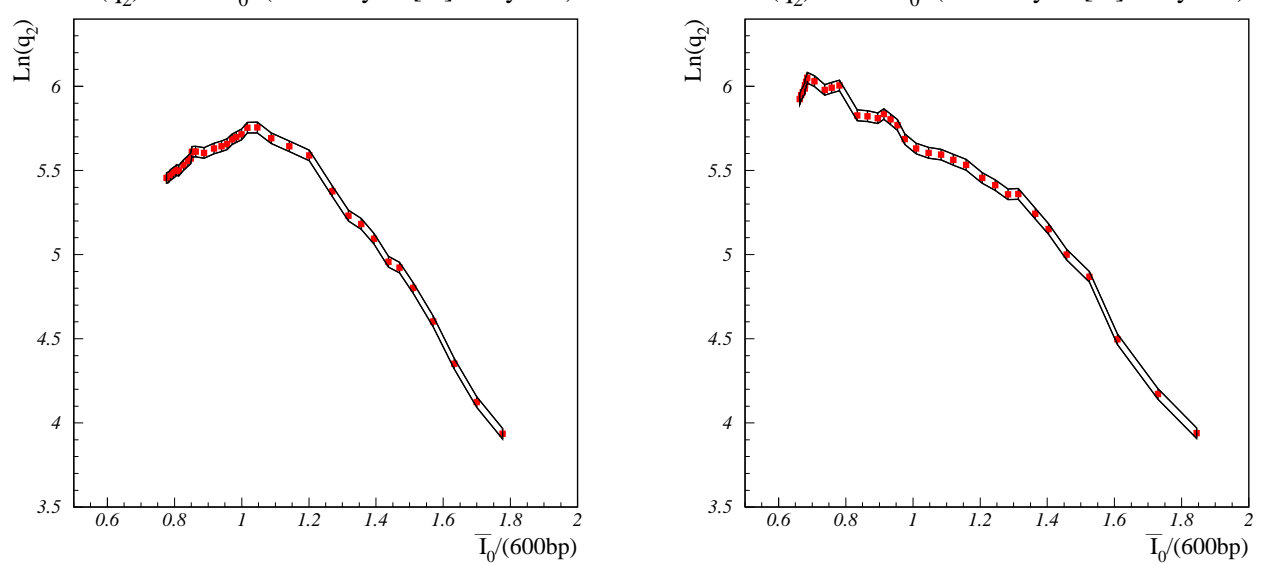

$\ln \left(\mathrm{q}_{2}\right)$ versus $\overline{\mathrm{I}}_{0}(\mathrm{~L}=1$ day $\&[\mathrm{~m}]=5$ years $)$
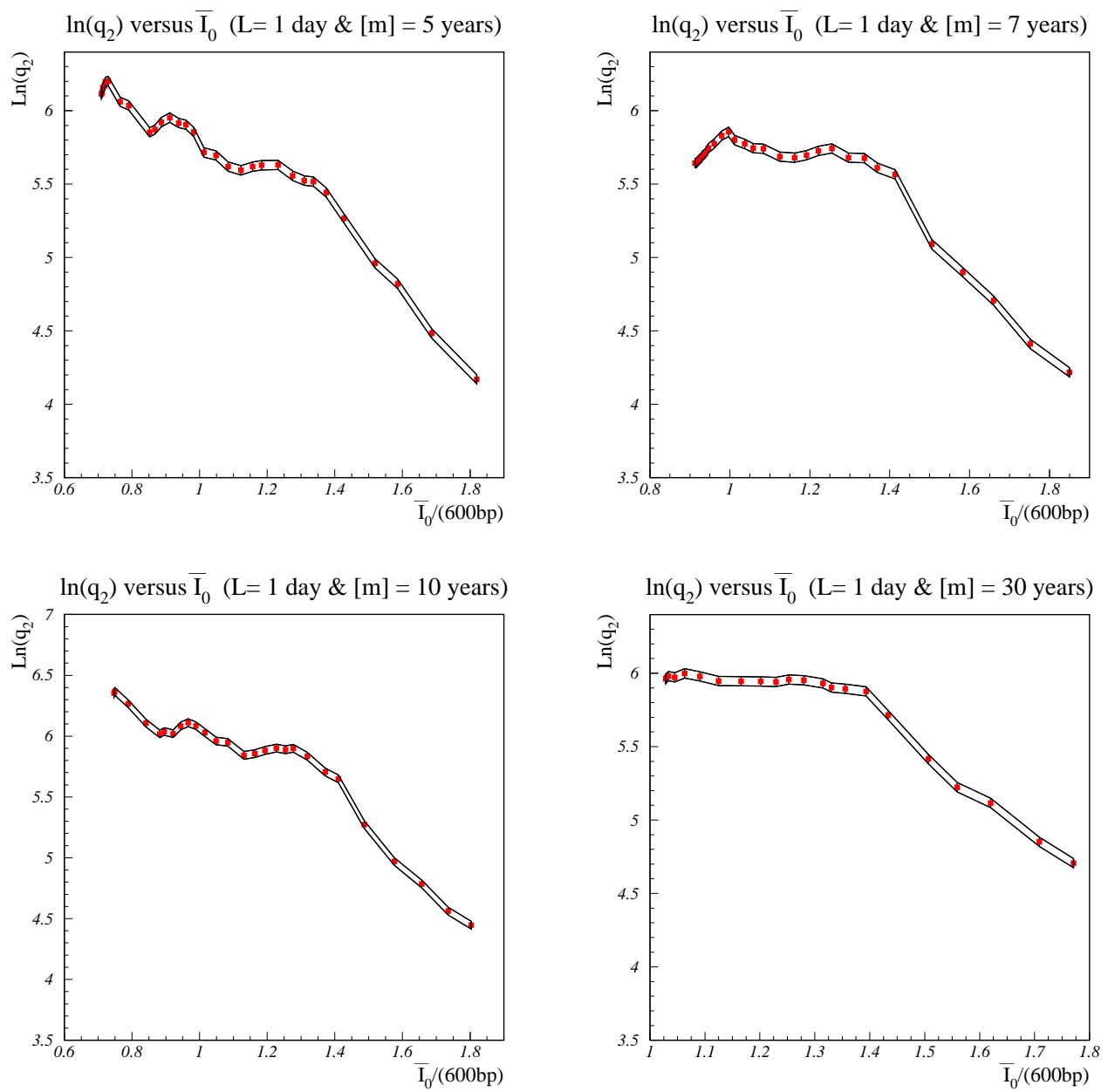
Figure (9)

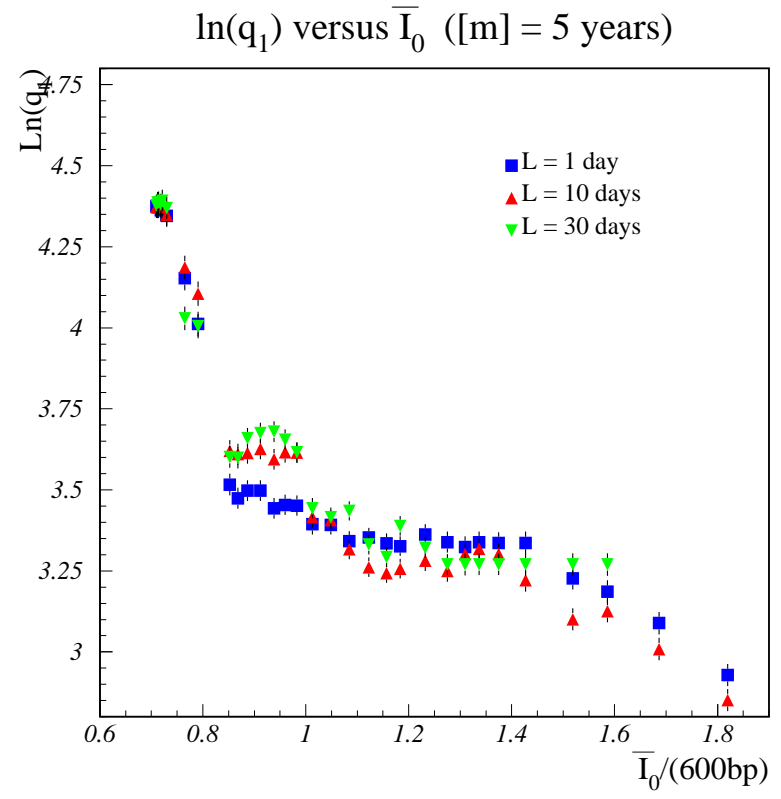

Figure (10)

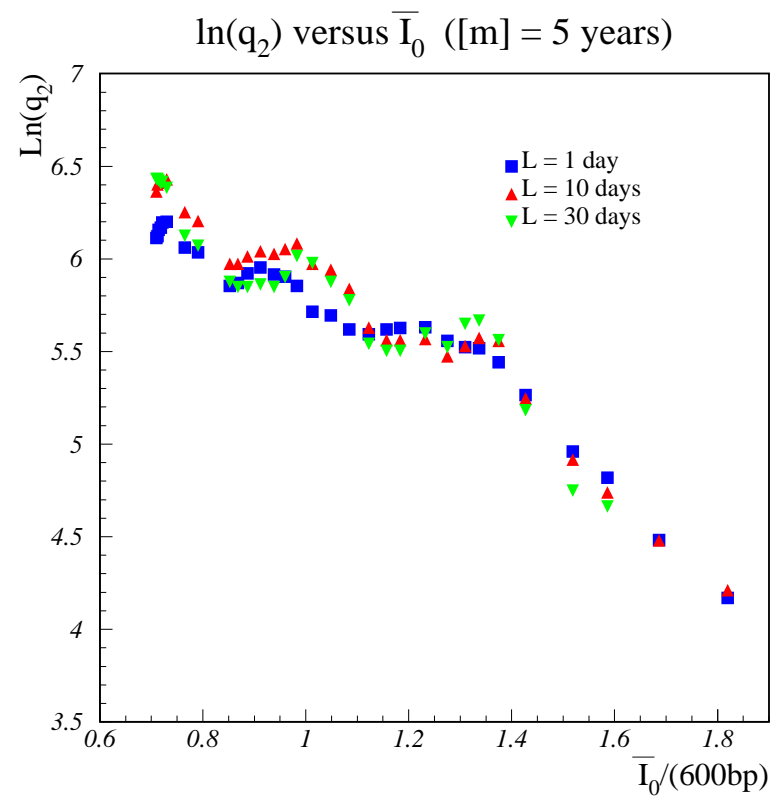


Table (1)

Data set $\{2\}$

\begin{tabular}{cccc}
\hline maturity $(\mathrm{yrs})$ & $\mu_{1}\left(\%^{-1}\right)$ & $\mu_{2}\left(\%^{-2}\right)$ & $\mu_{2} / \mu_{1}\left(\%^{-1}\right)$ \\
\hline 1 & $0.657 \pm 0.005$ & $1.197 \pm 0.005$ & $1.82 \pm 0.02$ \\
2 & $0.627 \pm 0.006$ & $1.178 \pm 0.007$ & $1.88 \pm 0.03$ \\
3 & $0.653 \pm 0.005$ & $1.164 \pm 0.006$ & $1.78 \pm 0.02$ \\
5 & $0.638 \pm 0.005$ & $1.139 \pm 0.005$ & $1.79 \pm 0.02$ \\
7 & $0.626 \pm 0.005$ & $1.111 \pm 0.005$ & $1.78 \pm 0.02$ \\
10 & $0.636 \pm 0.005$ & $1.100 \pm 0.005$ & $1.73 \pm 0.02$ \\
30 & $0.578 \pm 0.006$ & $1.068 \pm 0.006$ & $1.85 \pm 0.02$ \\
\hline
\end{tabular}

Table (2)

Data set $\{1\}$

\begin{tabular}{cccc}
\hline maturity (yrs) & $\mu_{1}\left(\%^{-1}\right)$ & $\mu_{2}\left(\%^{-2}\right)$ & $\mu_{2} / \mu_{1}\left(\%^{-1}\right)$ \\
\hline 1 & $0.607 \pm 0.006$ & $1.209 \pm 0.009$ & $1.99 \pm 0.03$ \\
2 & $0.644 \pm 0.004$ & $1.200 \pm 0.007$ & $1.86 \pm 0.03$ \\
3 & $0.652 \pm 0.004$ & $1.172 \pm 0.007$ & $1.80 \pm 0.02$ \\
5 & $0.622 \pm 0.006$ & $1.139 \pm 0.005$ & $1.83 \pm 0.02$ \\
7 & $0.606 \pm 0.006$ & $1.109 \pm 0.004$ & $1.83 \pm 0.02$ \\
10 & $0.597 \pm 0.005$ & $1.093 \pm 0.005$ & $1.83 \pm 0.02$ \\
30 & $0.588 \pm 0.005$ & $1.074 \pm 0.004$ & $1.83 \pm 0.02$ \\
\hline
\end{tabular}




\section{References}

[1] Nuyts, J. and Platten, I., Physica A 299 (2001) 528-546.

[2] Mandelbrot, B.B., Journal of Business, 36 (1963) 394-419.

[3] Mandelbrot, B.B., Fractals and Scaling in Finance, Springer (1997).

[4] Weron, R., Lévy-stable distributions revisited : tail index $>2$ does not exclude the Lévy-stable regime, cond-mat/0103256, Int. J. Modern Physics C, Vol. 12, No. 2 (2001) 209-223.

[5] Federal Reserve Statistics. Historical data. http://www.federalreserve.gov/releases/h15/data.htm.

[6] Di Matteo, T and Aste, T, How does the Eurodollar Interest Rate behave?, cond-mat/0101009, Journal of Theoretical and Applied Finance, 5 (2002) p.127122.

[7] Bouchaud, J-Ph, Power-laws in economy and finance: some ideas from physics, cond-mat/0008103, Proceedings of the Santa-Fe Conference 'Beyond Efficiency' held in May 2000, Journal of Quantitative Finance (to be published).

[8] Alderweireld, T and Nuyts, J., A Theory for the Term Structure of Interest Rates. (to be published).

[9] Fama, E. E., Journal of Business, 38 (1965) 35;

French, K.R., Journal of Financial Economics, 8 (1989) 55.

[10] Hill, B., Annals of Statistics, 35 (1975) 1163-1173. 


\title{
Detailed Empirical Study of the Term Structure of Interest Rates. Emergence of Power Laws and Scaling Laws
}

\author{
Thomas Alderweireld ${ }^{1}$ and Jean Nuyts ${ }^{2}$
}

\begin{abstract}
The technique of Padé Approximants, introduced in a previous work, is applied to extended recent data on the distribution of variations of interest rates compiled by the Federal Reserve System in the US. It is shown that new power laws and new scaling laws emerge for any maturity not only as a function of the Lag but also as a function of the average inital rate. This is especially true for the one year maturity where critical forms and critical exponents are obtained. This suggests future work in the direction of constructing a theory of variations of interest rates at a more "microscopic" level.
\end{abstract}

\footnotetext{
${ }^{1}$ Thomas.Alderweireld@umh.ac.be, Université de Mons-Hainaut, 20 Place du Parc, 7000 Mons, Belgium

2JJean.Nuyts@umh.ac.be, Université de Mons-Hainaut, 20 Place du Parc, 7000 Mons, Belgium
} 


\section{Introduction}

Many models have been constructed to try to describe and/or predict the term structure of interest rates. Recently, it has been shown on a specific set of data that the empirical distribution of the variation of interest rates is rather well reproduced by using the technique of Padé Approximants [1]. This article is a further step in this direction. The classical approach is based on Brownian motions of various kinds. It leads to exponential tails (or superposition of exponential tails) in the distributions. These so called narrow tails are usually badly rejected by the data. Levy-flight [2], [3], [4] distributions have also been proposed. In this case the tails of the distributions decrease as the inverse of a small power of the variation of the interest rates (see below (10)), in fact a power comprised between one and two. The predicted tails are really too fat and are also rejected by the data. Sometimes rather unjustified cuts have been used to sharpen the Levy tails.

In a recent paper [1], analyzing numerically the data (Data $\{1\}$ ) on the American daily spot interest rates compounded by the Federal Reserve System [5] between February 15, 1977 and August 4, 1997 ( $N_{\text {events }}=5108$ corresponding to 5108 opening days), it was discovered that the fat tails, known to appear in the empirical distributions, decrease essentially as a fourth power of the variation of the interest rate and are thus naturally amenable to ratios of polynomials. It was shown that the empirical distributions, averaged on the initial interest rates, are very well fitted with simple Padé Approximants. More recently, articles taking into account the fat, but not too fat, tails and power laws have been proposed [6]-[7].

In this paper, using more complete data (Data $\{2\}$ ) recently available, again from the FRS between January 2, 1962 and December 27, 2002 (10230 opening days, see (6) below), we have been able to study in a more detailed way the Padé fits. At a first stage, we confirmed the preceding analysis. The results are summarized in section 2. In section 3, we have refined the analysis by dividing the new data in suitable subsets, restricting ourselves to intervals in the initial interest rate. This has allowed us to confirm and to extend the validity of the preceding results, but also and more importantly to suggest new scaling laws.

These results will be used in a forthcoming article [8] to try to build a "microscopic model" of interest rates. 


\section{Padé Approximants fits. Update of the Previ- ous Results}

In this section, we present briefly the notation and update the results, which have been obtained in [1] for the Data $\{1\}$, using the full data set (Data $\{2\}$ ) available today [5].

Suppose that, at some initial time $t_{0}$, the interest rate (for some maturity $[\mathrm{m}]$ ) is $I_{0}$ and, at some later time $t_{f}$, it is $I_{f}$. The lag and the variation of interest rates were defined as follows

- The lag is the time interval between the initial and final times

$$
L=t_{f}-t_{0}
$$

It should be stressed that the lag is expressed in consecutive opening days and that non working days were simply discarded [9].

- The variation of interest rates is

$$
v=I_{f}-I_{0} .
$$

Since, in the data, the interest rates are expressed exactly in basis points (0.01 percent), the basis point has been chosen as the natural unit. When discretized, the variables $I$ and $v$ are thus represented by integers, $\widehat{I}$ and $\widehat{v}$.

Let us denote by $p^{[m]}\left(L, v, I_{0}\right)$ the probability distribution and by $\widehat{p}^{[m]}\left(L, \widehat{v}, \widehat{I}_{0}\right)$ the corresponding discretized distribution. They are the probability that the interest rate for maturity $[m]$ has moved from $I_{0}$ to $I_{f}$ during the lag $L$. Their norms are

$$
\begin{aligned}
\int_{-\infty}^{\infty} p^{[m]}\left(L, v, I_{0}\right) d v & =1 \\
\sum_{\widehat{v}=-\infty}^{\infty} \widehat{p}^{[m]}\left(L, \widehat{v}, \widehat{I}_{0}\right) & =1 .
\end{aligned}
$$

In the FRS Data $\{1\}$ and $\{2\}$, the maturities $[m]=1,2,3,5,7,10$ and 30 years are given. All the daily interest rates are expressed exactly in basis points. They vary roughly from $1.5 \%=150$ basis points to $18 \%=1800$ basis points. The discrete empirical distributions $\widehat{w}(\widehat{I})$ of the initial interest rates, for a given maturity 
and for the two sets, are defined by

$$
\begin{aligned}
& \widehat{w}(\widehat{I})=\frac{N^{d}(\widehat{I})}{N_{\text {events }}}, \\
& N^{d}(\widehat{I})=\text { number of days the interest rate, for maturity }[m] \text {, } \\
& \text { was } \widehat{I} \text { basis points in the FRS Data }\{1\} \text { or }\{2\} \text {, } \\
& N_{\text {events }}=5108 \text { in Data }\{1\} \text { for all }[m] \text {, } \\
& \begin{array}{|cc|c|c|c|c|c|c|c|}
\hline[m] & = & 1 & 2 & 3 & 5 & 7 & 10 & 30 \\
\hline N_{\text {events }}= & 10230 & 6606 & 10199 & 10199 & 8323 & 10199 & 6214 \\
\hline
\end{array}
\end{aligned}
$$

As an example, the FRS empirical distribution $\widehat{w}$ for the maturity $[m]=1$ year and for the Data $\{2\}$ is given in Figure (1). Obviously, we have for any such distribution

$$
\sum_{\widehat{I}=150}^{1800} \widehat{w}(\widehat{I})=1
$$

The main subject of study in [1] was in fact the discrete average $\widehat{p}_{\text {average }}^{[m]}(L, \widehat{v})$ defined by

$$
\widehat{p}_{\text {average }}^{[m]}(L, \widehat{v})=\sum_{\widehat{I}_{0}=150}^{1800} \widehat{w}^{[m]}\left(I_{0}\right) \widehat{p}^{[m]}\left(L, \widehat{v}, \widehat{I}_{0}\right) .
$$

The discretized averages can easily be extracted from the data for lags (in days) such that $1 \leq L \leq 30$ and for variations of interest rates $\widehat{v}$ (expressed in basis points) included in the range $R_{v}=[-200 \leq \widehat{v} \leq 200]$. Indeed, outside this range, the data is always zero. The discrete normalization becomes

$$
\sum_{\widehat{v}=-200}^{200} \widehat{p}_{\text {average }}^{[m]}(L, \widehat{v})=1 .
$$

It has been first shown empirically, using Data $\{1\}$, that the tail of the term structure of the interest rates decreases essentially as a fourth power of the variation of the interest rates i.e.

$$
p_{\text {average }}^{[m]}(L, v) \approx \kappa \frac{1}{|v|^{d}} \quad \text { when }|v| \text { becomes large }
$$

with a suitable constant $\kappa$ and a power $d$

$$
d \approx 4
$$


This means that the distributions decrease much faster than what Levy flight models suggest (namely $1 \leq d \leq 2$ ) but much more slowly than what Brownian models $p_{\text {average }} \approx \kappa \exp \left(-v^{2} / \sigma^{2}\right)$ predict.

Using detailed fits, it has been shown in [1] that the average distribution (8) can be very well (amazingly well) approximated by a Padé Approximant $P(0,4)$, i.e. with a constant numerator and a polynomial of fourth degree in the denominator. More precisely, for all maturities $[m]$ and for all lags $L$, the following Padé form of the probability distributions, in terms of $\widehat{v}$,

$$
\widehat{p}_{\text {average }}^{[m]}(L, \widehat{v})=\frac{q_{1}}{\pi\left(1+\left(q_{1}^{2}+2 q_{2}\right) \widehat{v}^{2}+q_{2}^{2} \widehat{v}^{4}\right)},
$$

fits the Data $\{2\}$ (and the Data $\{1\}$ set) rather well.

Let us enumerate a few of the properties of this form and of the two remaining real parameters $q_{1}$ and $q_{2}$ which depend on the lag $L$ and on the maturity $[m]$.

1. The continuous distribution corresponding to (12) is, for $q_{1}$ positive, a purely positive real function. In fact it is constructed as the modulus square of the complex function $f$

$$
\begin{aligned}
f_{\text {average }}^{[m]}(L, v) & =\frac{\sqrt{q_{1}}}{\sqrt{\pi}\left(1+i q_{1} v+q_{2} v^{2}\right)}, \\
p_{\text {average }}^{[m]}(L, v) & =\left|f_{\text {average }}^{[m]}(L, v)\right|^{2} .
\end{aligned}
$$

2. The normalization (3) of the distribution (14) is automatic by construction.

3. The distribution depends only on $v^{2}$, hence it is symmetrical under the interchange $\{v \leftrightarrow-v\}$. This symmetry was checked empirically to hold to a very precise degree. In other words, violations of this symmetry are zero well within one standard deviation.

4. The tail of the distribution (12) is of the form $v^{-4}$. The Bayesian Hill estimators $[10]$ of the empirical tails favor an approximate decrease of this kind.

5. An estimate of $q_{1}$ is given by

$$
q_{1}=\pi \widehat{p}_{\text {average }}^{[m]}(L, 0)
$$

and of $q_{2}$ by

$$
q_{2}=-\sigma^{-1}
$$

where $\sigma$ stands for the variance of the data distribution. 
6. The parameters $q_{1}$ and $q_{2}$ have very simple regular forms in term of the lag $L$ when $L$ varies from one day to about a month and for any maturity $[m]$. Essentially

$$
q_{i} \approx \beta_{i}\left(\frac{L}{L_{\mathrm{r}}}\right)^{-\mu_{i}} \quad, \quad i=1,2
$$

where, for convenience, we have chosen one arbitrary reference value $L_{\mathrm{r}}=$ 15 days. The $\mu_{i}$, which we call scaling parameters, and the less essential multiplication constants $\beta_{i}$ depend on $[m]$ but not on $L$. The Table (1) (resp. Table (2)) shows the $\mu_{i}$ and the ratio $\mu_{2} / \mu_{1}$ that we have obtained for the 7 investigated maturities using the Data $\{2\}$ (resp. Data $\{1\}$ ) set.

In other words, as illustrated by the Figures (2) and (3) for $[m]=2$ years, as an example, in a $\ln \left(q_{i}\right)$ versus $\ln (L)$ plot the data points align along a straight line with slope $-\mu_{i}$. The plots for the other $[m]$ 's follow analogous straight lines.

7. Moreover, one may note that one critical exponent is approximatively related to the other exponent by (see Tables (1) (2))

$$
\mu_{2} \approx 1.8 \mu_{1}
$$

8. It should be stressed that this scaling law (17) seems to be an important discovery [1] which had escaped attention.

\section{Padé Approximants fits. Initial Interest Rate Dependence}

More recently, we have studied the extended set of data (Data $\{2\}$ ) and performed a more refined analysis.

Indeed, the new data having often a higher statistics, meaningful subsets of data can be defined with initial interest rates limited to intervals. We have found that subsets containing about two thousand events are large enough to lead to a sufficiently precise determination of the parameters.

A detailed numerical analysis of the distributions restricting the data to an almost fixed initial interest rate $I_{0}$ has convinced us that the same Padé behavior prevails. An approximate determination of the dependence of the Padé parameters $q_{i}$ on initial interest rate $I_{0}$ follows. It suggests that new scaling laws are at work.

Let us summarize our results 
1. We have first divided the Data $\{2\}$ set in subsets where $I_{0}$ is restricted to regions (overlapping or not)

$$
\Delta=\left[\widehat{I}_{0 \min }, \widehat{I}_{0 \max }\right] .
$$

The total probability $\omega_{\Delta}$ to be inside $\Delta$ and the total numbers of events $N_{\Delta}$ inside $\Delta$ are given by

$$
\begin{aligned}
\omega_{\Delta} & =\sum_{\widehat{I}=\widehat{I}_{0 \min }}^{\widehat{I}_{\text {max }}} \widehat{w}(\widehat{I}), \\
N_{\Delta} & =\omega_{\Delta} N_{\text {events }} .
\end{aligned}
$$

In the present analysis, the regions have be chosen in such a way that the $N_{\Delta}$ 's are around two thousands. If the number of events is chosen to be smaller than about two thousand, the accuracy in the determination of the parameters becomes hazardous. On the other hand, there seems to be no need to select larger regions with more events in them. The events in Data $\{2\}$ can thus, for example for $[m]=1$ year, be separated in five non overlapping intervals covering the full $\widehat{I}_{0}$ range.

2. Let us define $\overline{I_{0}}$ as the empirical average of $\widehat{I}$ in a given region $\Delta$

$$
\overline{I_{0}}=\frac{\sum_{\widehat{I}=\widehat{I}_{0 \min }}^{\widehat{I}_{\max }} \widehat{w}(\widehat{I}) \widehat{I}}{\omega_{\Delta}} .
$$

3. The distributions, for the events within a region $\Delta$ (see (24) for a precise definition), are then supposed, in first approximation, to be represented by normalized (3) Padé forms

$$
\bar{p}\left(L, \widehat{v}, \overline{I_{0}}\right)=\frac{q_{1}}{\pi\left(1+\left(q_{1}^{2}+2 q_{2}\right) \widehat{v}^{2}+q_{2}^{2} \widehat{v}^{4}\right)} .
$$

4. The parameters $q_{i}$ appearing in Eq.(23) (for a given maturity $[m]$ ) are thus evaluated as functions of $L$ and $\overline{I_{0}}$. They are obtained from the data by minimizing a $\chi^{2}$ function. In about all instances the fits are very good as exemplified by the $\chi^{2}$ values.

5. In Figure (4), as an example using the Data $\{2\}$ with $L=1$ and $[m]=1$ year, two thousand points in the region $\Delta=[420$ basis points, 553 basis points] 
(see (19)) have been selected and the empirical points are plotted versus the best Padé fit. The average initial interest rate $(22)$ is about $\overline{I_{0}}=502$ basis points. In this case, the values of $q_{i}$ evaluated in their respective units are $q_{1}=38.6 \pm 1.3(\%)^{-1}$ and $q_{2}=-308 \pm 19(\%)^{-2}$. The obtained $\chi^{2}$ is 61 with 83 degrees of freedom. In almost all $L,[\mathrm{~m}]$ cases an eye check of the curves is as satisfactory and the computed $\chi^{2}$ are as good.

6. At this point, an important remark is worth making. It should be stressed that the parameters $q_{i}$ obtained this way are not really those at $I_{0}=\overline{I_{0}}$ itself but a complicated average of the approximate $q_{i}$ in the corresponding $\Delta$ range. Indeed, it is obvious that the superposition of two or more Padé forms is, at best, only approximatively of a Padé form. Since this is an important point, let us explain it more precisely. If $\widehat{p}(L, \widehat{v}, \widehat{I})$ is known for any $\widehat{I}$, then the average probability $\bar{p}\left(L, \widehat{v}, \overline{I_{0}}\right)(23)$ on $\Delta$ is defined by

$$
\bar{p}\left(L, \widehat{v}, \overline{I_{0}}\right)=\frac{\sum_{\widehat{I}=\widehat{I}_{0 \min }}^{\widehat{I_{\max }}} \widehat{w}(\widehat{I}) \widehat{p}(L, \widehat{v}, \widehat{I})}{\omega_{\Delta}} .
$$

If it then were true that $\widehat{p}(L, \widehat{v}, \widehat{I})$ is exactly of the Padé form (which is anyhow not a correct statement but, at best, an approximate one), the $\Delta$-average probability $\bar{p}\left(L, \widehat{v}, \overline{I_{0}}\right)$ would not exactly be of a Padé form.

7. After the fits have been performed, we find that the new parameters $q_{i}$ depend effectively and importantly on $\overline{I_{0}}$.

8. In a first approach, we have chosen to minimize the natural $\chi^{2}$ leaving $q_{1}$ as free parameter and by determining $q_{2}$ from the experimental variance using the relation (16). The interested reader can find the precise definition of the $\chi^{2}$ in the reference [1], in and after their Eq.(22).

9. We defer to the next section a detailed discussion of the parameters $q_{i}$, obtained from the data, as functions of the lag $L$ and of the average interest rate $\bar{I}_{0}$.

\section{Padé parameters as a function of the lag, of the maturity and, of the initial interest rate}

In this section, we summarize the scaling law and the $\bar{I}_{0}$ dependence that are suggested by the empirical data. 


\subsection{General considerations and scaling laws}

The first scaling law and the $I_{0}$ dependence can be summarized as follows. For each maturity, the empirical $q_{i}$ are given by the approximate forms (compare to (17))

$$
q_{i} \approx \beta_{i}\left(\frac{L}{L_{\mathrm{r}}}\right)^{-\mu_{i}} \mathcal{F}_{i}\left(\overline{\overline{I_{0}}} \overline{I_{r}}\right) .
$$

where the $\mathcal{F}_{i}$ are functions depending of the initial interest rate. For convenience, we have chosen two arbitrary reference values

$$
\begin{aligned}
L_{\mathrm{r}} & =15 \text { days } \\
I_{\mathrm{r}} & =600 \text { basis points } .
\end{aligned}
$$

In $(25)$, the lag $L$ is in days, the average initial rate $\bar{I}_{0}$ is in basis points, the parameters $q_{1}$ and $\beta_{1}$ are in inverse basis points, the parameters $q_{2}$ and $\beta_{2}$ are in inverse basis points squared while the scaling parameters $\mu_{i}$ are pure numbers.

The scaling law (25) says that, for every maturity $[m]$, each of the parameters $q_{i}$ is the product of two functions. One function depends on the lag $L$ only and has the form of a power law as (17). The second function, $\mathcal{F}_{i}$, depends on $\bar{I}_{0}$ only.

The best values for the parameters $\mu_{i}$ are given in Table 1 as a function of the maturity. The form of the $\mathcal{F}_{i}$ can be found in the fourteen plots given in Figures (5)(8). In Figure (5), for $[m]=1$ year and $L=1$ day, the $\ln \left(q_{1}\right)$ is plotted versus $\bar{I}_{0} / I_{r}$. In Figure (6), for $[m]=1$ year and $L=1$ day, the $\ln \left(q_{2}\right)$ is plotted versus $\bar{I}_{0} / I_{r}$. In Figure $(7)$ there are six plots where for $L=1, \ln \left(q_{1}\right)$ is given versus $\bar{I}_{0} / I_{r}$ for $[m]=$ 2, 3, 5, 7, 10, 30 years. Finally, in Figure (8) there are six plots where for $L=1$, $\ln \left(q_{2}\right)$ is given versus $\bar{I}_{0} / I_{r}$ for $[m]=2,3,5,7,10,30$ years.

We have shown that the assumption in (25) that the $q_{i}$ factorize (separation of variables) is favoured by the data. Hence, for a given maturity, the distributions of

$\ln \left(q_{i}\right)$ as a function of $\bar{I}_{0}$ for different $L$ values just differ by a constant shift. This shift on the $\ln \left(q_{i}\right)$ axis is equal to

$$
\ln \left(q_{i}\right)-\ln \left(q_{i}^{\prime}\right)=\mu_{i} \ln \left(L^{\prime} / L\right) .
$$

To illustrate this, the Figures (9) and (10) show the distributions of $\ln \left(q_{i}\right)$, for $[m]=5$ years as a function of $\bar{I}_{0}$ for 3 different $L$ values (1, 10 and 30 days). To make the comparison easier, the $L=10$ days and the $L=30$ days distributions have been moved upwards by their respective shift term $\left(\mu_{i} \ln (10)\right.$ and $\mu_{i} \ln (30)$ respectively). For the other maturity, one obtains similar plots.

It has to be emphasized that this variable separation in the approximate forms may be a hint of a scaling law which is at work. 


\subsection{The scaling laws for the $[m]=1$ year case}

For the $[m]>1$ year data, no particular simple shape for the function $\mathcal{F}_{i}$ can be extracted. In the $[m]=1$ year data, the functions $\mathcal{F}_{i}$ have a very simple shape. The

$\ln \left(q_{i}\right)$ distributions as function of $\overline{I_{0}}$ (see Figures (5)(6) for $L=1$ day) can be fitted with straight lines and the following extremely simple approximate forms hold

$$
q_{i} \approx \beta_{i}\left(\frac{L}{L_{\mathrm{r}}}\right)^{-\mu_{i}} \exp \left(\left(\frac{\overline{I_{0}}}{I_{r}}\right)^{-\nu_{i}}\right)
$$

where

$$
\begin{gathered}
\ln \left(\beta_{1}\right)=3.14 \pm 0.02, \quad \mu_{1}=0.657 \pm 0.005, \quad \nu_{1}=1.32 \pm 0.02 \\
\ln \left(\beta_{2}\right)=4.51 \pm 0.02, \quad \mu_{2}=1.197 \pm 0.005, \quad \nu_{2}=2.19 \pm 0.02
\end{gathered}
$$

Hence, for the particular case of $[m]=1$ year, we obtain an interplay of two simple scaling laws with critical exponents $\mu_{i}$ and $\nu_{i}$.

\section{Conclusion}

In this paper, we have extended an earlier work aimed at approximating the term structure of interest rates by ratios of polynomials called Padé Approximants. The form which is used is a purely positive Padé $P[0,4]$ with a constant numerator and a fourth degree denominator in the variation of interest rates.

We have shown that for any maturity, for any lag and for initial interest rates restricted to regions, the form holds to a very good degree of approximation, better than what should have been hoped a priori.

The empirical parameters are represented by functions which point toward the existence of scaling laws and scaling exponents at a more "microscopic level". These scaling laws were already discovered and discussed [1] in terms the lag variable. In this paper, using the extended FRS data [5] now available, we have shown that scaling laws seem also to be at work not only in the lag, as was shown previously, but also in the initial interest rate. This is true especially for $[m]=1$ year maturity where simple critical forms (28) appear both in the lag $L$ and in the average interest rate $\bar{I}_{0}$ and critical exponents can be extracted.

In a forthcoming paper, we will try to use this result and the scaling laws which are suggested by the data to build a theory of the term structure of interest rates at a "microscopic level". 


\section{Acknowledgment}

The work of one author (T.A.) was supported in part by Belgian I.I.S.N. (Institut Interuniversitaire des Sciences Nucléaires). The work of the other (J.N.) was supported in part by the Belgian F.N.R.S. (Fonds National de la Recherche Scientifique). He would also like to thank Pr. Isabelle Platten for discussions and numerical simulations in an early phase of this research. 


\section{Figure Caption}

Figure 1.

The FRS empirical weight distribution $\widehat{w}$ of the interest rates (see (5)) for the maturity $[m]=1$ year and for the Data set $\{2\}$.

Figure 2 .

Distribution of $\ln \left(q_{1}\right)$ as a function of $\ln (L / 15)$ for $[m]=2$ years using Data set $\{2\}$.

Figure 3 .

Distribution of $\ln \left(q_{2}\right)$ as a function of $\ln (L / 15)$ for $[m]=2$ years using Data set $\{2\}$.

Figure 4 .

One example of a fit to the Data set $\{2\}$ for $[m]=1$ year and $L=1$ day by a Padé Approximant (continuous curve). The chosen subset (see (19)) corresponds to 2000 points in the region $I_{0 \min }=420$ and $I_{0 \max }=553$. The $\bar{I}_{0}$ is computed to be 502 basis points. The empirical points are plotted versus the best Padé fit. In this case, the values of $q_{i}$ evaluated in their respective units are $q_{1}=38.6 \pm 1.3(\%)^{-1}$ and $q_{2}=-308 \pm 19(\%)^{-2}$. The obtained $\chi^{2}$ is 61 with 83 degrees of freedom.

\section{Figure 5.}

The parameter $\ln \left(q_{1}\right)$ as a function of $\overline{I_{0}} / 600$ for $[m]=1$ year and $L=1$ day. These points have been obtained by minimizing the $\chi^{2}$ as a function of parameter $q_{1}$. Each point corresponds to a subset of data containing close to two thousand points with average $I_{0}$ value equal to $\overline{I_{0}}$. The subsets are overlapping so that the points are not independent. The unit used for $q_{1}$ is $(\%)^{-1}$. For $\overline{I_{0}}$ it is the basis point. The straight line is a linear fit. Its equation is $\ln \left(q_{1}\right)=\ln \left(\beta_{1}\right)-\nu_{1}\left(\overline{I_{0}} / 600\right)-\mu_{1} \ln (1 / 15)$ with $\nu_{1}=1.32 \pm 0.02$ and $\ln \left(\beta_{1}\right)=3.14 \pm 0.02$.

Figure 6. 
The parameter $\ln \left(q_{2}\right)$ as a function of $\overline{I_{0}} / 600$ for $[m]=1$ year and $L=1$ day. These points have been obtained using the relation defined in (16). Each point corresponds to a subset of data containing close to two thousand points with average $I_{0}$ value equal to $\overline{I_{0}}$. The subsets are overlapping so that the points are not independent. The error on $\ln \left(q_{2}\right)$ is computed by propagating the error on the estimate of the variance. The unit used for $q_{2}$ is $(\%)^{-2}$. For $\overline{I_{0}}$ it is the basis point. The straight line is a linear fit. Its equation is $\ln \left(q_{2}\right)=$ $\ln \left(\beta_{2}\right)-\nu_{2}\left(\overline{I_{0}} / 600\right)-\mu_{2} \ln (1 / 15)$ with $\nu_{2}=2.19 \pm 0.02$ and $\ln \left(\beta_{2}\right)=4.51 \pm 0.02$.

\section{Figure 7.}

The parameter $\ln \left(q_{1}\right)$ as a function of $\overline{I_{0}} / 600$ for $[m]=2,3,5,7,10,30$ years and $L=1$ day. These points have been obtained by minimizing the $\chi^{2}$ as a function of parameter $q_{1}$. Each point corresponds to a subset of data containing close to two thousand points with average $I_{0}$ value equal to $\overline{I_{0}}$. The subsets are overlapping so that the points are not independent. The unit used for $q_{1}$ is $(\%)^{-1}$. For $\overline{I_{0}}$ it is the basis point.

\section{Figure 8.}

The parameter $\ln \left(q_{2}\right)$ as a function of $\overline{I_{0}} / 600$ for $[m]=2,3,5,7,10,30$ years and $L=1$ day. These points have been obtained using the relation (16). Each point corresponds to a subset of data containing close to two thousand points with average $I_{0}$ value equal to $\overline{I_{0}}$. The subsets are overlapping so that the points are not independent. The unit used for $q_{2}$ is $(\%)^{-2}$. For $\overline{I_{0}}$ it is the basis point. The error on $\ln \left(q_{2}\right)$ is computed by propagating the error on the estimate of the variance.

Figure 9 .

Distribution of $\ln \left(q_{1}\right)$, for $[m]=5$ years as a function of $\overline{I_{0}} / 600$ for 3 different $L$ values (1,10 and 30 days) where the $L=10$ and 30 days distributions have been shifted on the $\ln \left(q_{1}\right)$ axis by the correcting term $(27)$.

Figure 10.

Distribution of $\ln \left(q_{2}\right)$, for $[m]=5$ years as a function of $\overline{I_{0}} / 600$ for 3 different $L$ values (1,10 and 30 days) where the $L=10$ and 30 days distributions have been shifted on the $\ln \left(q_{2}\right)$ axis by the correcting term $(27)$. 


\section{Table Caption}

Table 1.

The values of the best fits to the parameters $\mu_{i}$ (see (25)) using Data set $\{2\}$. These parameters are given as a function of the maturity $[m]$. The standard errors correspond to a unit deviation in the $\chi^{2}$.

Table 2 .

The values of the best fits to the parameters $\mu_{i}$ (see (25)) using Data set $\{1\}$. These parameters are given as a function of the maturity $[m]$. The standard errors correspond to a unit deviation in the $\chi^{2}$. 
Figure (1)

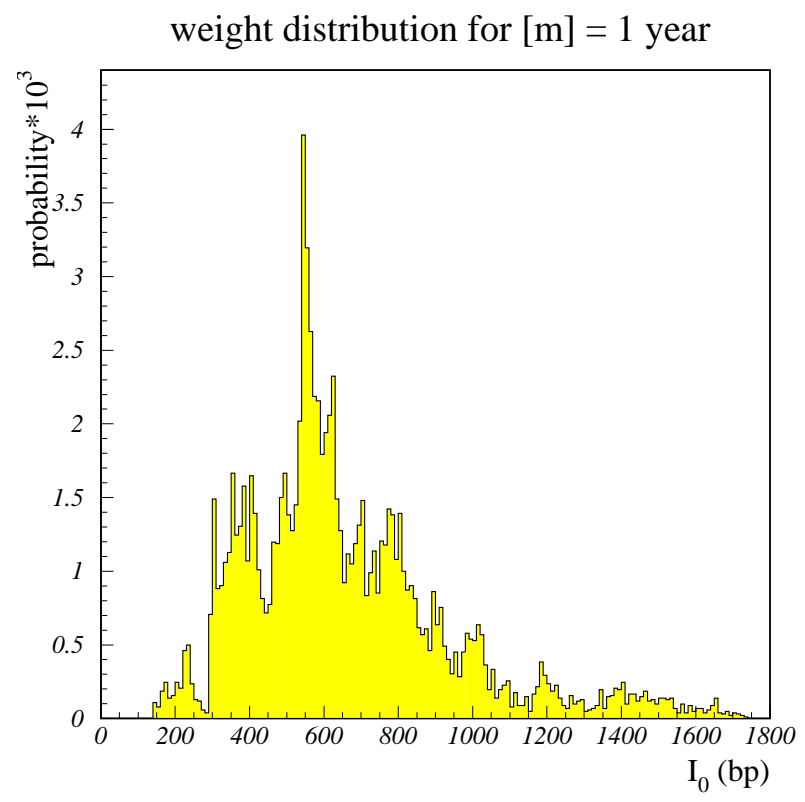

Figure (2)

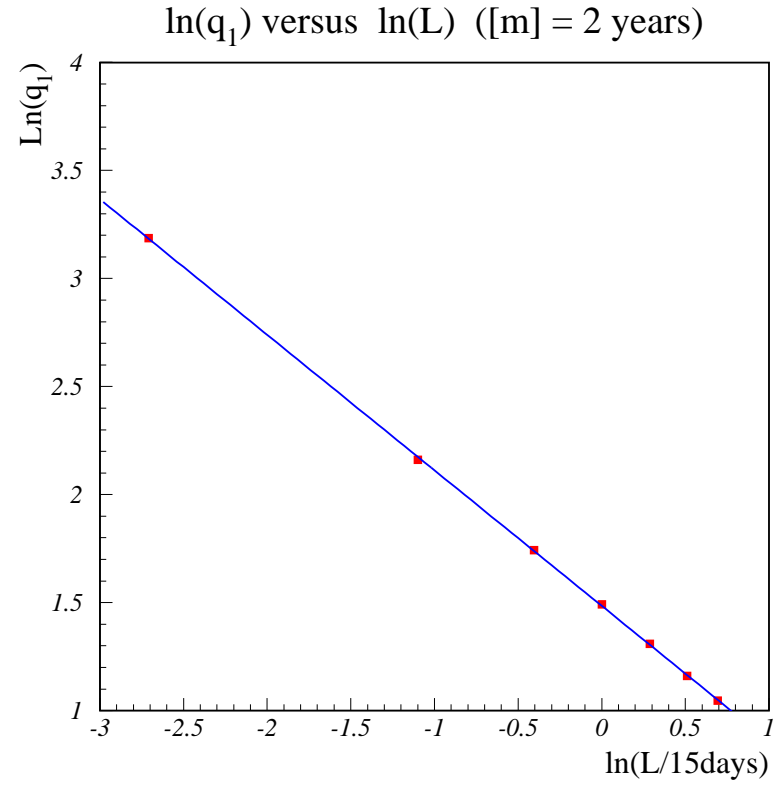


Figure (3)

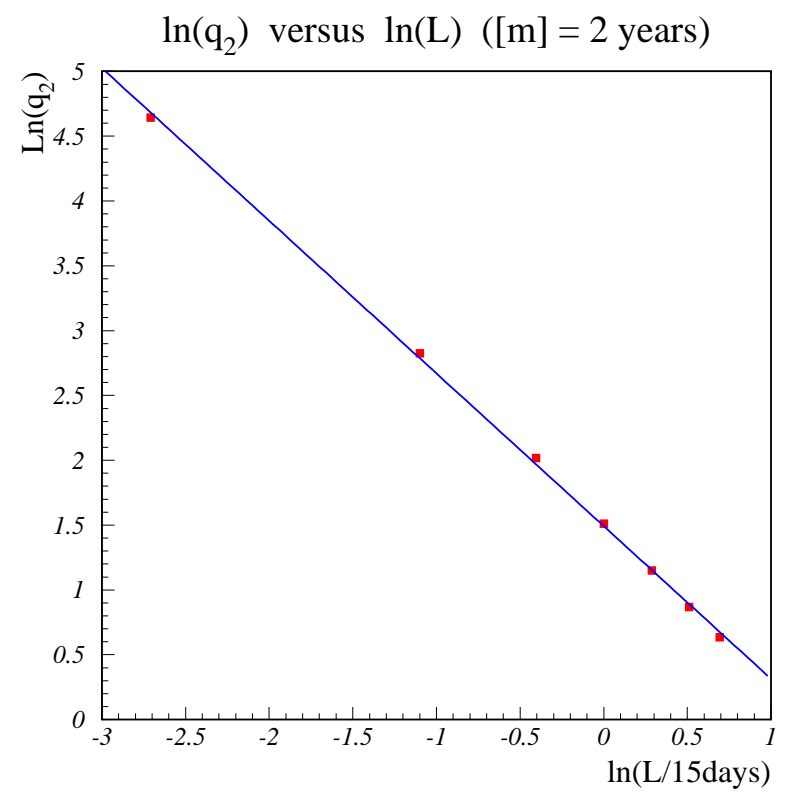

Figure (4)

A fit for $[\mathrm{m}]=1$ year, $\mathrm{L}=1$ day and average $\mathrm{I}_{0}=502 \mathrm{bp}$

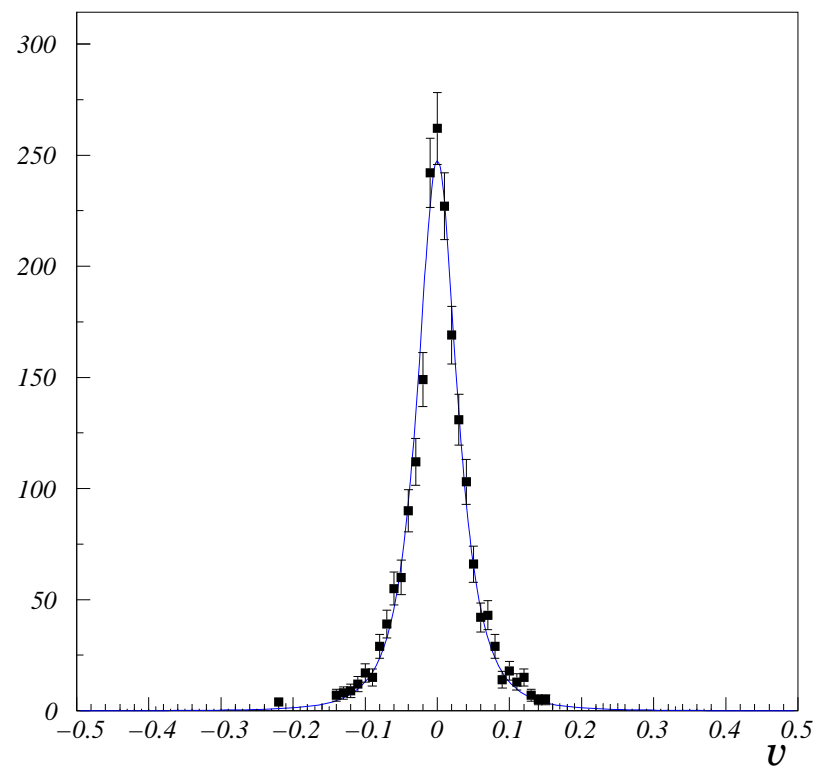


Figure (5)

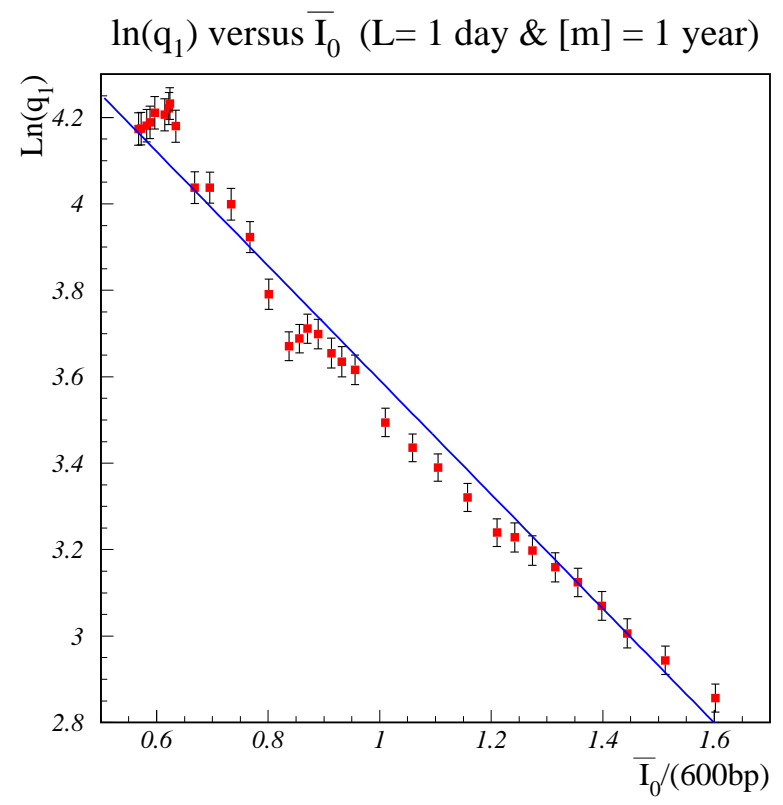

Figure (6)

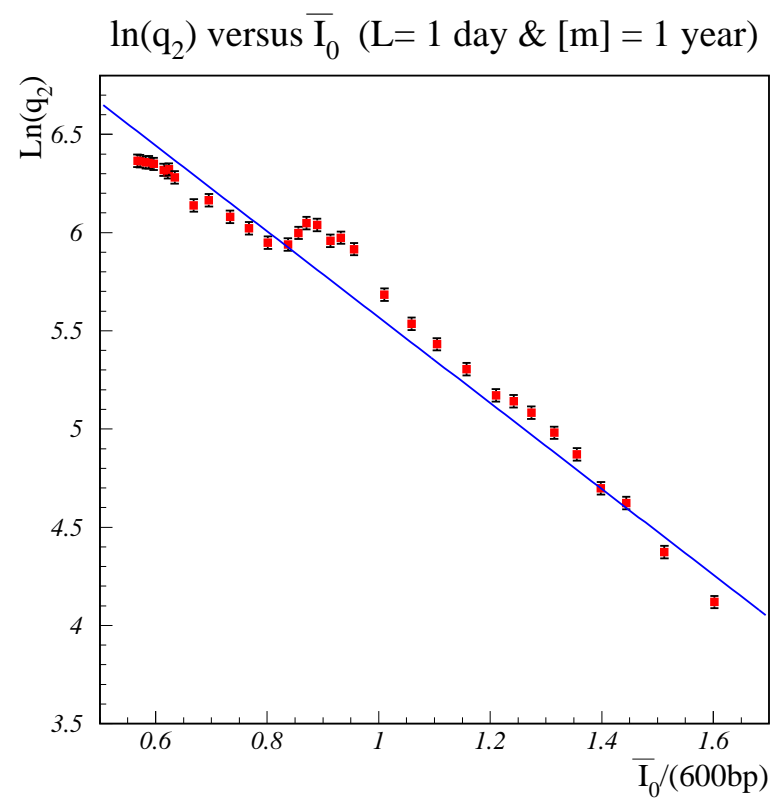


Figure (7)
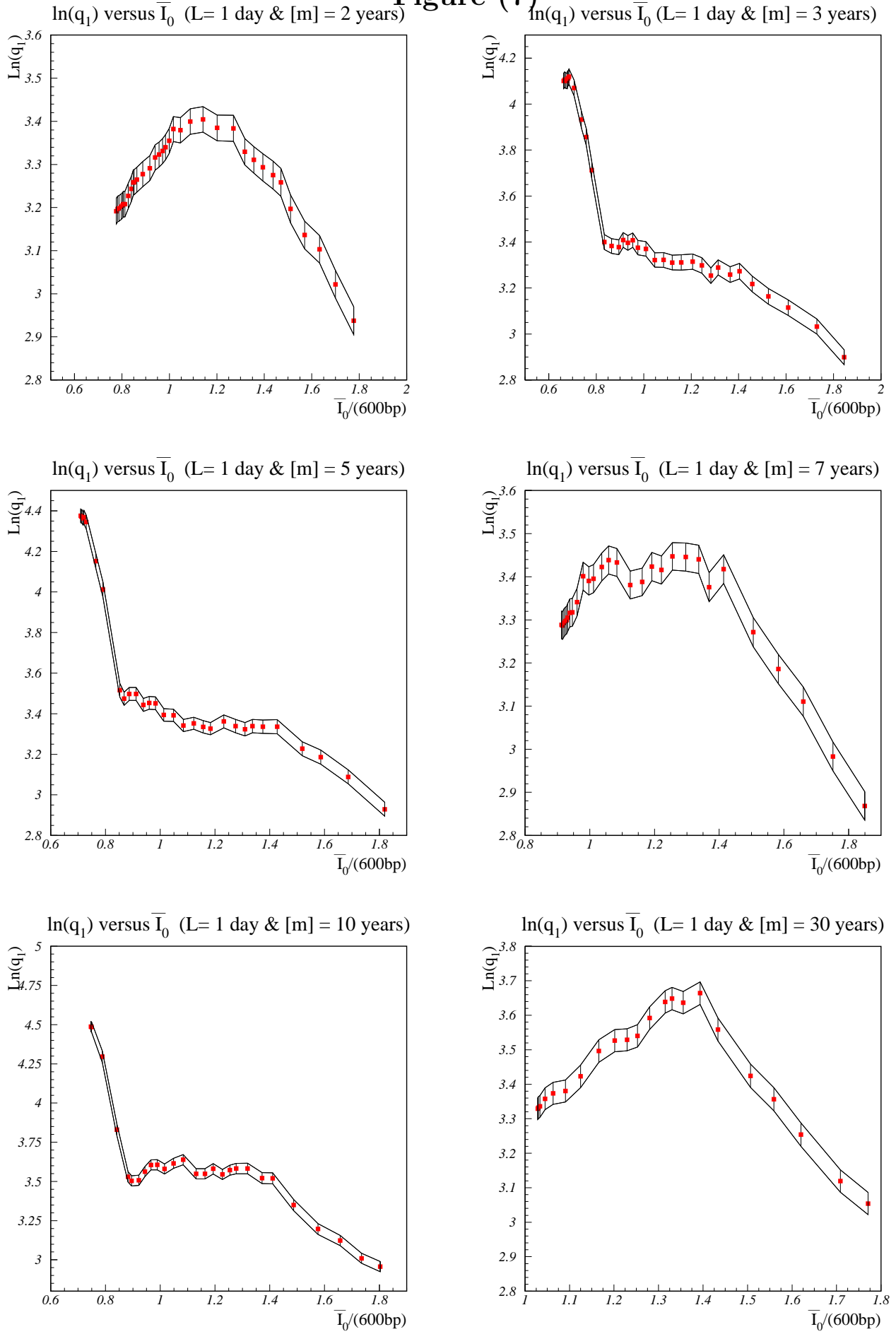
Figure (8)
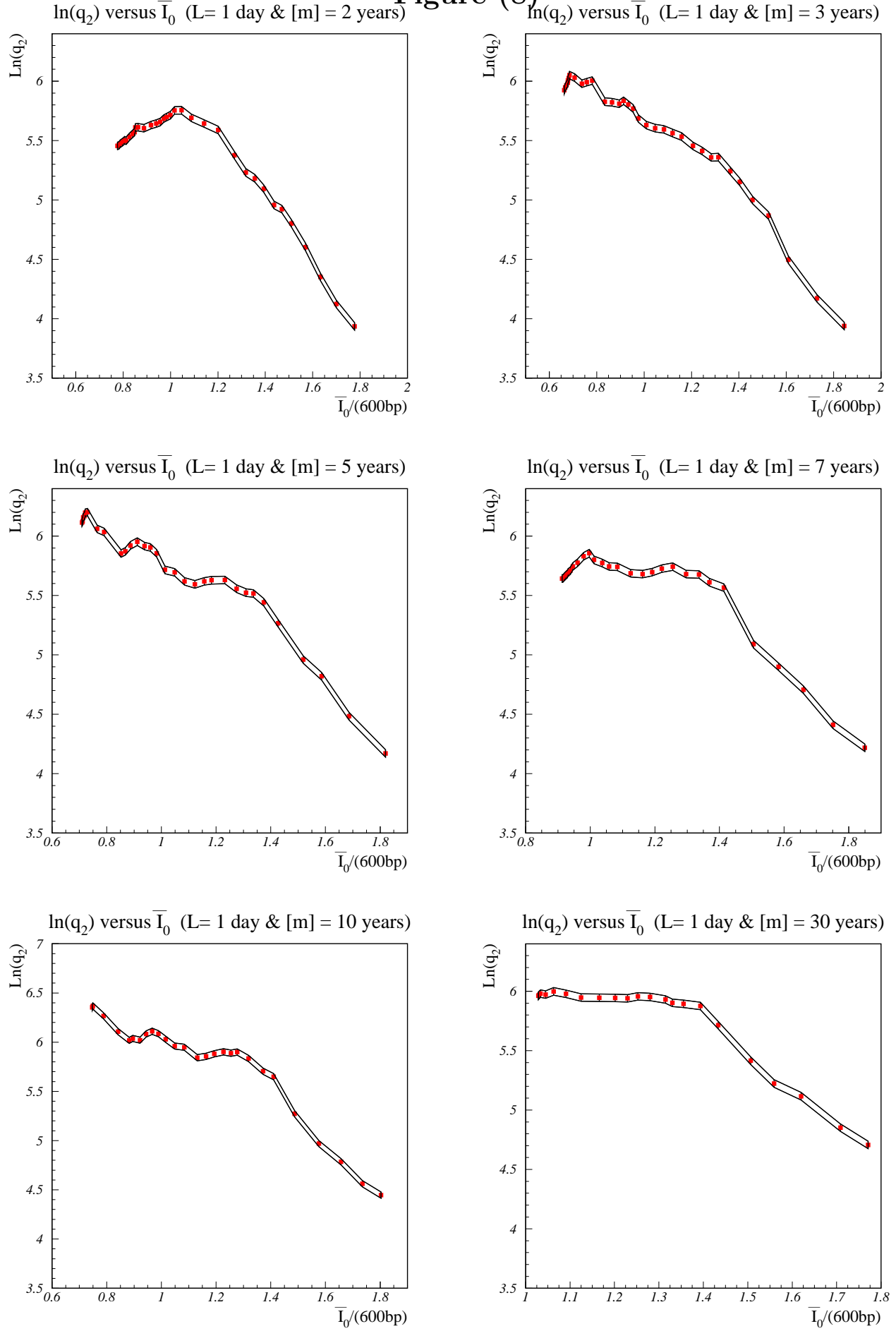
Figure (9)

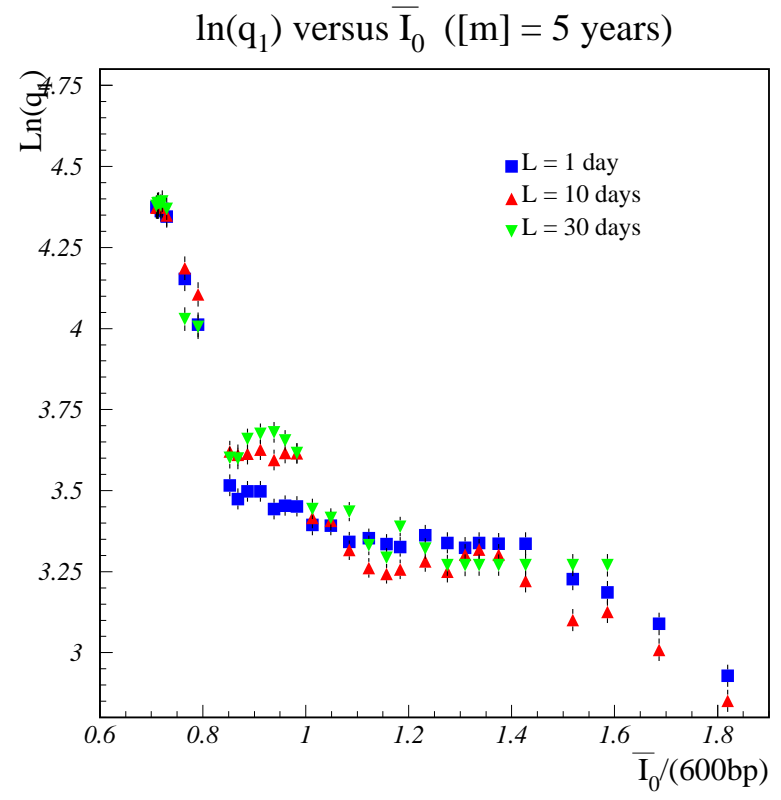

Figure (10)

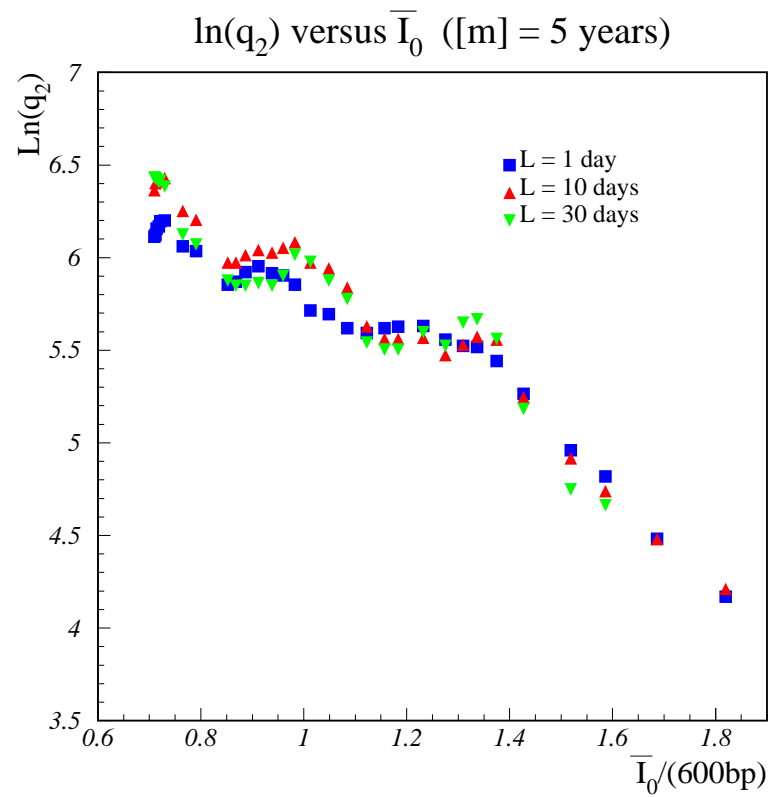




\section{Table (1)}

Data set $\{2\}$

\begin{tabular}{cccc}
\hline maturity (yrs) & $\mu_{1}\left(\%^{-1}\right)$ & $\mu_{2}\left(\%^{-2}\right)$ & $\mu_{2} / \mu_{1}\left(\%^{-1}\right)$ \\
\hline 1 & $0.657 \pm 0.005$ & $1.197 \pm 0.005$ & $1.82 \pm 0.02$ \\
2 & $0.627 \pm 0.006$ & $1.178 \pm 0.007$ & $1.88 \pm 0.03$ \\
3 & $0.653 \pm 0.005$ & $1.164 \pm 0.006$ & $1.78 \pm 0.02$ \\
5 & $0.638 \pm 0.005$ & $1.139 \pm 0.005$ & $1.79 \pm 0.02$ \\
7 & $0.626 \pm 0.005$ & $1.111 \pm 0.005$ & $1.78 \pm 0.02$ \\
10 & $0.636 \pm 0.005$ & $1.100 \pm 0.005$ & $1.73 \pm 0.02$ \\
30 & $0.578 \pm 0.006$ & $1.068 \pm 0.006$ & $1.85 \pm 0.02$ \\
\hline
\end{tabular}

Table (2)

Data set $\{1\}$

\begin{tabular}{cccc}
\hline maturity $(\mathrm{yrs})$ & $\mu_{1}\left(\%^{-1}\right)$ & $\mu_{2}\left(\%^{-2}\right)$ & $\mu_{2} / \mu_{1}\left(\%^{-1}\right)$ \\
\hline 1 & $0.607 \pm 0.006$ & $1.209 \pm 0.009$ & $1.99 \pm 0.03$ \\
2 & $0.644 \pm 0.004$ & $1.200 \pm 0.007$ & $1.86 \pm 0.03$ \\
3 & $0.652 \pm 0.004$ & $1.172 \pm 0.007$ & $1.80 \pm 0.02$ \\
5 & $0.622 \pm 0.006$ & $1.139 \pm 0.005$ & $1.83 \pm 0.02$ \\
7 & $0.606 \pm 0.006$ & $1.109 \pm 0.004$ & $1.83 \pm 0.02$ \\
10 & $0.597 \pm 0.005$ & $1.093 \pm 0.005$ & $1.83 \pm 0.02$ \\
30 & $0.588 \pm 0.005$ & $1.074 \pm 0.004$ & $1.83 \pm 0.02$ \\
\hline
\end{tabular}




\section{References}

[1] Nuyts, J. and Platten, I., Physica A 299 (2001) 528-546.

[2] Mandelbrot, B.B., Journal of Business, 36 (1963) 394-419.

[3] Mandelbrot, B.B., Fractals and Scaling in Finance, Springer (1997).

[4] Weron, R., Lévy-stable distributions revisited : tail index $>2$ does not exclude the Lévy-stable regime, cond-mat/0103256, Int. J. Modern Physics C, Vol. 12, No. 2 (2001) 209-223.

[5] Federal Reserve Statistics. Historical data. http://www.federalreserve.gov/releases/h15/data.htm.

[6] Di Matteo, T and Aste, T, How does the Eurodollar Interest Rate behave?, condmat/0101009, Journal of Theoretical and Applied Finance, 5 (2002) p.127-122.

[7] Bouchaud, J-Ph, Power-laws in economy and finance: some ideas from physics, cond-mat/0008103, Proceedings of the Santa-Fe Conference 'Beyond Efficiency' held in May 2000, Journal of Quantitative Finance (to be published).

[8] Alderweireld, T and Nuyts, J., A Theory for the Term Structure of Interest Rates. (to be published).

[9] Fama, E. E., Journal of Business, 38 (1965) 35;

French, K.R., Journal of Financial Economics, 8 (1989) 55.

[10] Hill, B., Annals of Statistics, 35 (1975) 1163-1173. 


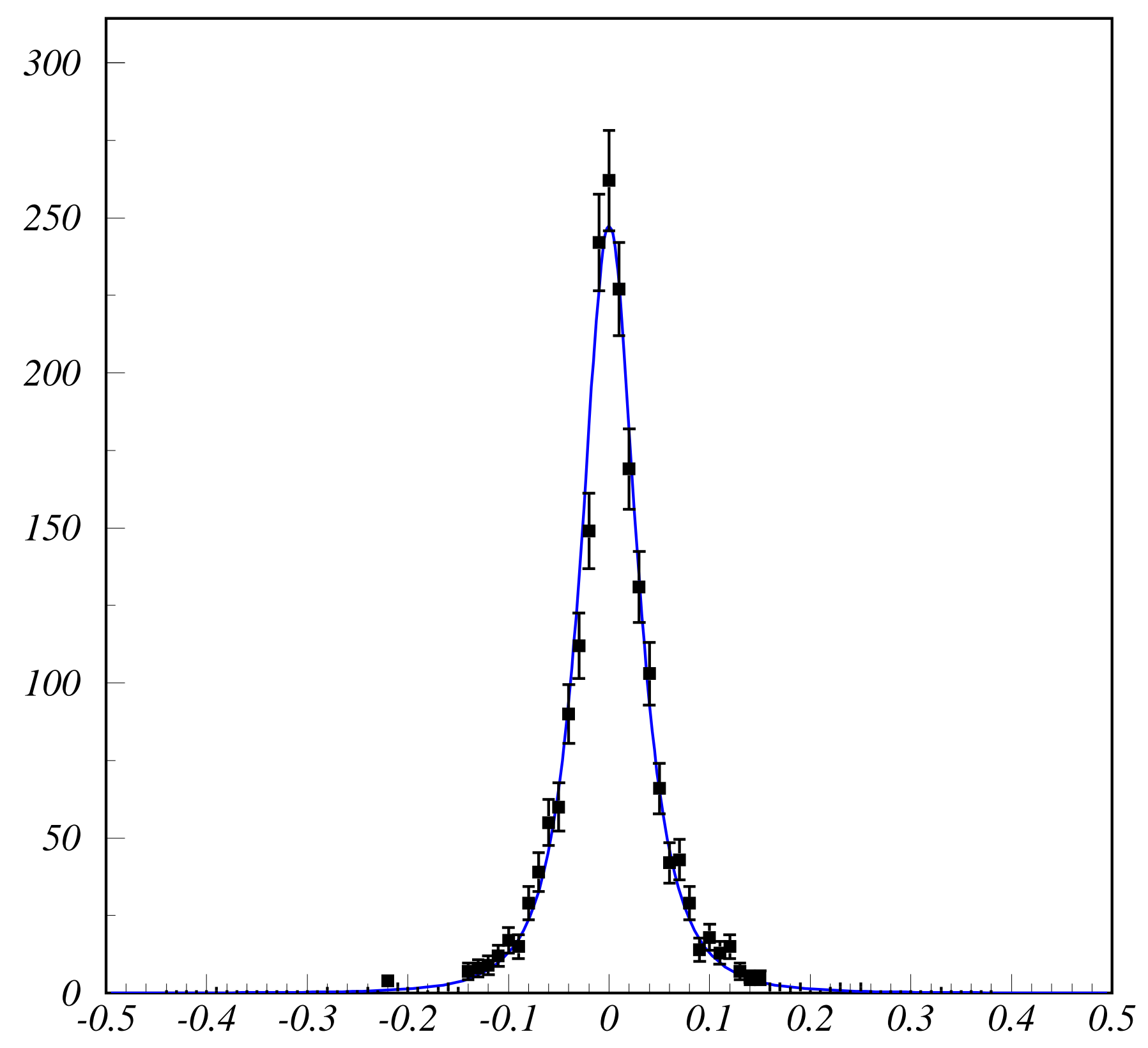

